\title{
Il Programma Globale delle NaZioni Unite CONTRO LA TRATTA DI ESSERI UMANI
}

\author{
Margherita BLANDINI* \\ INVESTIGADORA, DERECHO INTERNACIONAL PÚBLICO \\ UNIVERSIDAD DE LA RIOJA
}

\section{s u m a r i o}

I. Introduzione. I. Una fattispecie criminale di dimensioni incerte. 2. Il ruolo dell'united nations office on drugs and crime. II. Il sistema normativo di riferimento per l'azione delle nazioni unite. I. Definizione internazionalmente riconosciuta della fattispecie di tratta. 2. Armonizzazione delle legislazioni. III. Sviluppo di una strategia a livello nazionale. IV. Cooperazione internazionale nel campo della giustizia penale. I. Estradizione. 2. Reciproca assistenza legale. 3. Altre forme di cooperazione. 4. Metodi d'indagine. V. Assistenza e protezione di vittime e testimoni. I. Identificazione delle vittime. 2. Ambiti di protezione. 3. Status di residenza, rimpatrio e reintegrazione. 4. Restituzioni e risarcimento per le vittime. VI. Prevenzione del fenomeno di tratta. VII. Progetti di assistenza tecnica. VIII. Monitoraggio e valutazione dei progetti e dei programmi. IX. Valutazione del Programma Globale. X. Conclusioni.

\section{Introduzione}

I. Una fattispecie criminale di dimensioni incerte

La tratta di esseri umani ha raggiunto, negli ultimi decenni, proporzioni enormi; nessun paese può essere considerato immune da quest'attività criminale. Parallelamente, vi è stato un boom di informazioni sul fenomeno: nuovi articoli, libri, reports e manuali vengono pubblicati settimanalmente, ma l'affidabilità dei contenuti di tali pubblicazioni

\footnotetext{
* Questa ricerca è frutto della collaborazione temporanea prestata dall'autrice, in qualitá di intern, presso l'Anti-Human Trafficking Unit dell' United Nations Office on Drugs and Crime di Vienna.

Le opinioni espresse sono dell'autrice e non possono considerarsi come il riflesso della posizione dell'Anti-Human Trafficking Unit dell' United Nations Office on Drugs and Crime di Vienna.
} 
rimane un problema irrisolto. Ancora oggi non si dispone di dati precisi ed univoci, a testimonianza di come sia piuttosto deficitaria una conoscenza organica del fenomeno sia da parte degli Stati che da parte delle strutture nazionali ed internazionali chiamate ad occuparsi della lotta contro tale tipo di attività criminale. Oltre che su dati forniti dagli apparati investigativi nazionali ed internazionali, l'analisi della tratta di persone si basa attualmente su una pluralità di stime elaborate da diversi enti internazionali e da organizzazioni non governative. Queste stime presentano, in alcuni casi, valori molto diversi tra loro e sono spesso utilizzate al fine della raccolta di fondi. Pertanto, se da un lato esse denotano un pregevole sforzo finalizzato alla riduzione dell'incertezza conoscitiva sulle dimensioni e le dinamiche della tratta, dall'altro, impongono a ciascuno di accostarsi alla loro lettura in forma critica, dato che non sempre sono esplicitati i criteri di rilevazione e le fonti dalle quali provengono i dati forniti. Sono queste le ragioni che hanno spinto l' United Nations Office on Drugs and Crime (UNODC) a promuovere, all'interno del Programma Globale contro la Tratta di esseri umani, un progetto mirato all'elaborazione di un global database sui diversi aspetti della tratta di esseri umani. Il database contiene dati quantitativi e qualitativi comparabili tra Paesi e tra Regioni del Mondo e divisi in tre principali sezioni: country reports, profili delle vittime di tratta e dei trafficanti, rotte della tratta ${ }^{2}$.

Le Nazioni Unite hanno iniziato ad occuparsi della tratta di esseri umani sin dalla propria fondazione, anzi si può dire che la protezione dei diritti umani e l'abolizione di ogni forma di schiavitù costituiscono due dei principali obiettivi intorno ai quali sono state costruite le Nazioni Unite al termine dell'ultimo conflitto mondiales. Lo sforzo della comunità internazionale si è sempre concentrato sulla tutela delle vittime di tratta contro la violazione dei loro diritti umani. In conseguenza, le norme internazionali sui diritti umani

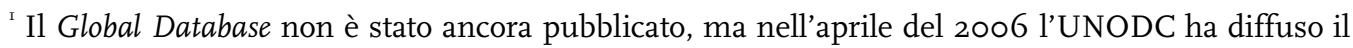
Report «Trafficking in Persons: Global Patterns», che anticipa parte del suo contenuto, <http://www.unodc.org/pdf/traffickinginpersons_report_2006ver2.pdf> [consultato: 03-I2-2007].

Per un'analisi critica dei risultati preliminari emersi nel corso dell'elaborazione del Global Database si veda KANGASPUNTA, K., «Mapping the inhuman trade: preliminary findings of the database on trafficking in human beings» in Forum on Crime and Society, Vol. 3, Num. I and 2, December 2003.

${ }^{2}$ La comparabilità, tuttavia, non sempre è possibile; sotto il profilo della legislazione, ad esempio, non in tutti i Paesi esiste una normativa specifica sulla tratta e dunque non vi sono statistiche sul numero dei casi di tratta nonché dei soggetti inquisiti e condannati ed, anche laddove una specifica normativa esiste, le definizioni legali della fattispecie variano da un Paese ad un altro e dunque le previsioni possono risultare più o meno ampie. Inoltre, in alcuni Paesi, non vi è una chiara distinzione tra le ipotesi di trafficking e quelle di smuggling o, anche dove il legislatore abbia distinto le fattispecie, a livello di investigazione da parte delle forze di polizia e durante tale fase, la distinzione non sussiste.

${ }^{3}$ E' quanto esplicitato nella Carta delle Nazioni Unite, firmata a San Francisco il 26 giugno del I945 ed il cui articolo I recita: «I fini delle Nazioni Unite sono: I. Mantenere la pace e la sicurezza internazionale, ed a questo fine: prendere efficaci misure collettive per prevenire e rimuovere le minacce alla pace e per reprimere gli atti di aggressione o le altre violazioni della pace e conseguire con mezzi pacifici, ed in conformità ai princìpi della giustizia e del diritto internazionale, la composizione o la soluzione delle controversie o delle situazioni internazionali che potrebbero portare ad una violazione della pace. 2. Sviluppare tra le nazioni relazioni amichevoli fondate sul rispetto e sul principio dell'eguaglianza dei diritti e dell'auto-decisione dei popoli e prendere altre misure atte a rafforzare la pace universale; 3. Conseguire la cooperazione internazionale nella soluzione dei problemi internazionali di carattere economico, sociale culturale od umanitario, e nel promuovere ed incoraggiare il rispetto dei diritti dell'uomo e delle libertà fondamentali per tutti senza distinzioni di razza, di sesso, di lingua o di religione; 4. Costituire un centro per il coordinamento dell'attività delle nazioni volta al conseguimento di questi fini comuni».
} 
e le consuetudini internazionali esistenti consentono di estrapolare alcuni standards di protezione delle vittime che gli Stati sono tenuti a rispettare ${ }^{4}$. Essi sono:

- conformità delle legislazioni nazionali ad un'unica definizione di tratta di persone;

- principio di non discriminazione delle vittime nelle leggi sostanziali e procedurali nonché nell'investigazione e nella pratica;

- diritto alla sicurezza e ad un giusto trattamento;

- diritto di accesso alla giustizia;

- diritto ad ottenere il risarcimento del torto subito;

- diritto allo status di residente;

- diritto alla salute ed agli altri servizi sociali;

- diritto al rimpatrio ed alla reintegrazione;

- principio di cooperazione tra gli Stati.

Tali forme di protezione devono essere garantite a tutte le persone trafficate, a prescindere che si tratti di donne, uomini o bambini. Gli standards prevedono un incentivo per le vittime che decidono di denunciare il reato alle autorità e di agire in qualità di testimoni.

Il collegamento tra la tratta di esseri umani e la rete della criminalità organizzata è una questione relativamente recente. Capire come si è sviluppato un mercato di dimensioni mondiali e come vengono organizzati i flussi illeciti è la nuova prospettiva adottata dalle Nazioni Unite nella lotta contro questo fenomeno. I gruppi criminali organizzati sono in continua ricerca di nuove opportunità, essi si dirigono laddove esiste una domanda ed i rischi sono bassi. La domanda di attraversamento illegale delle frontiere è cresciuta negli ultimi decenni e, dato che le sanzioni per questo tipo di attività in molti Paesi sono lievi (se comparate, ad esempio, con quelle previste per il traffico di droga), o addirittura inesistenti, e che il livello di corruzione è alto, il business è apparso conveniente agli occhi del crimine organizzato 5 .

Come già discusso in precedenza, a causa del carattere di illegalità e clandestinità del fenomeno risulta molto difficile fornire dati precisi sulle sue dimensioni e, pertanto, bisogna valutare con le dovute cautele quei dati di cui disponiamo che, anche se non completamente affidabili, sono comunque indicativi dei preoccupanti effetti provocati dal fenomeno e costituiscono la ragione per cui la Comunità Internazionale si è resa conto della necessità di adoperare strumenti nuovi e più adeguati per contrastarlo, secondo la più

${ }^{4}$ Per maggiori approfondimenti si veda Global Alliance Against Trafficking In Women, FOUNDATION AGAINST TRAFFICKING IN WOMEN, INTERNATIONAL HuMAN RightS LAW GROUP, Human Rights Standards for the treatment of trafficked persons, versione aggiornata al 2005, <http://gaatw.net/Spanish texts/Normas version 2005.pdf> [consultato: 03-I2-2007].

${ }^{5}$ Sull'argomento si veda InTERnAtional CEntre For Migration POlicy Development, The Relationship between Organised Crime and Trafficking in Aliens, Vienna: ICMPD, June I999. 
aggiornata prospettiva che lo considera una forma di crimine organizzato oltre che una violazione dei diritti umani delle persone trafficate ${ }^{6}$.

\section{Il ruolo dell' United Nations Office on Drugs and Crime (UNODC)}

La globalizzazione crea nuove minacce da parte del crimine organizzato, cui i Governi devono far fronte. Al fine di assistere gli Stati in queste difficili sfide, l'UNODC ha riunito alcune squadre di specialisti che lavorano per sconfiggere una serie identificata di problemi globali. Si parla, per questa ragione, di Programmi Globali, facendo riferimento con tale espressione all'azione di entità specializzate impegnate a realizzare progetti di cooperazione tecnica di alta qualità ${ }^{7}$. Le squadre di esperti comprendono sia staff regolare delle Nazioni Unite sia altro personale specializzato, reclutato mediante private contribuzioni. Gli esperti, traendo lezione dall'esperienza raccolta sul terreno e dalle registrate tendenze generali del fenomeno, sono chiamati a disegnare le best practices, che devono poi essere diffuse tra tutti gli operatori a livello internazionale. La raccolta dei dati è continua e le risultanze di essa devono essere rese note attraverso manuali, toolkits, riviste ed Internet. L'assistenza tecnica fornita mediante i Programmi Globali ha lo scopo di rendere le forze nazionali di ciascun Paese autonome e capaci di mantenere il rispetto della legge; ciò viene realizzato mediante servizi di assistenza legale, training delle forze di polizia, degli avvocati e dei giudici, consigli sulle misure di prevenzione, supporto alle vittime ed ai testimoni. L'obiettivo di tali Programmi è di dare attuazione al Piano d'Azione fissato dagli Stati membri ed espresso all'interno delle Convezioni e dei Protocolli delle Nazioni Unite ${ }^{8}$.

Il Global Programme against Trafficking in human beings (GPAT) è stato lanciato nel marzo del I999 su iniziativa dell'UNODC di Vienna, in collaborazione con l'United Nations Interregional Crime and Justice Research Institute (UNICRI) di Torino. Esso, partendo da una prospettiva che focalizza l'approccio al fenomeno sulla componente di giustizia penale dello stesso, si propone di assistere gli Stati membri nella prevenzione e nel tentativo d'eliminazione della tratta, nonché nell'assistenza e nella protezione delle vittime. Il GPAT viene portato avanti dall'UNODC in collaborazione con altre Agenzie delle Nazioni Unite, tra cui, a parte l'UNICRI, principalmente l'International Organization for Migration (IOM),

\footnotetext{
${ }^{6}$ Per un'analisi dei dati, si veda U.S. DePARTMENT OF STATE, Trafficking in persons Report, June 2005, http://www.state.gov/documents/organization/47255.pdf [consultato: 03-I2-2007]. Secondo le stime del Governo degli Stati Uniti, il numero di esseri umani trafficati ogni anno nel mondo è compreso fra 600.000 ed 800.000 e la tratta di esseri umani genera un profitto di 9,5 miliardi di dollari all'anno (costituendo il terzo mercato per importanza dopo quello di armi e droga). BELSER, P., Forced Labour and Human Trafficking: estimating the profits, 2005,

<http://www.ilo.org/wcmsp5/groups/public/---ed_norm/--declaration/documents/publication/wcms_08I97I.pdf> [consultato: 03-I2-2007].

Secondo l'International Labour Organisation (ILO) i profitti derivanti dallo sfruttamento del lavoro forzato corrispondono a US\$44 billion l'anno e quelli derivanti dallo sfruttamento sessuale US\$27.8 billion.

${ }^{7}$ Per un'analisi più approfondita si veda <http://www.unodc.org/pdf/crime/publications/cicp_global_programmes.pdf> [consultato: 03-I22007].

${ }^{8}$ I sei Programmi Globali previsti sono dedicati, infatti, all'attuazione della Convenzione contro il Traffico Illecito di Sostanze Stupefacenti e Psicotrope (I988), della Convenzione sul Crimine Transnazionale Organizzato (2003), del Protocollo sulla Tratta di Persone (2003), delle previsioni contro il riciclaggio del denaro sporco contenute nelle Convenzioni su Droghe e Crimine, della Convenzione contro la Corruzione (2005) e delle Convenzioni contro il Terrorismo.
} 
l'United Nations Children's Fund (UNICEF), l'United Nations Development Programme (UNDP), e con Organizzazioni non Governative, tra cui, per citarne alcune, Save the Children, La Strada e Caritas.

Punti chiave del GPAT sono la raccolta di dati rilevanti riguardo alla diffusione della tratta nei vari Paesi, l'analisi e la valutazione delle misure anti-tratta esistenti nei vari Paesi, i progetti di cooperazione tecnica e lo sviluppo di meccanismi di coordinamento a livello nazionale ed internazionale.

Il punto di partenza è costituito da un'intensa attività di ricerca volta a valutare i flussi della tratta e la struttura dei gruppi organizzati che la promuovono, nonché a raccogliere dati sulle rotte, sulle modalità del trasporto e del successivo sfruttamento delle vittime. Tale attività è stata e continua ad essere compiuta nei Paesi d'origine, transito e destinazione del fenomeno mediante analisi d'ogni fonte disponibile, compresi casi giudiziari ed interviste alle vittime, ad operatori di giustizia, a rappresentanti delle agenzie che controllano l'immigrazione, ad operatori sociali e ad altri tecnici attivi nelle istituzioni locali e nelle organizzazioni non governative.

Le attività di cooperazione tecnica sono svolte in alcuni Paesi dell'Asia, dell'Europa, dell'Africa e del Latino America selezionati sulla base dei risultati delle analisi svolte e tenendo conto della gravità del fenomeno e del tipo di problemi connessi. Nella scelta dei Paesi è determinante l'impegno politico dei Governi e la misura in cui il Paese prescelto potrà essere utilizzato come modello di riferimento in una certa Regione del mondo. Particolare attenzione è riservata a Paesi con economie in transizione ed in via di sviluppo.

Tra le attività previste, a livello nazionale, sono comprese la promozione di campagne d'informazione per accrescere la coscienza sul fenomeno, l'assistenza legale per l'introduzione di normative adeguate, misure d'institution-building, la creazione di strutture di sostegno per vittime e testimoni, corsi di formazione per gruppi professionali (funzionari di polizia, delle dogane, degli apparati di giustizia e dei servizi sociali). A livello internazionale, il Programma mira a fornire assistenza alle Agenzie, alle Istituzioni locali ed ai Governi affinché lo sforzo congiunto di queste diverse forze porti all'elaborazione di misure più efficaci per la sconfitta del fenomeno.

L’UNODC non è, in realtà, una vera e propria Agenzia specializzata dell'ONU, ma piuttosto (al pari dell'UNICEF e dell'UNDP) un Programma, istituito con lo scopo di fornire ai Governi e ad altre Istituzioni, assistenza tecnica e finanziaria per la risoluzione di problemi internazionali, quali in questo caso quelli del Crimine Internazionale e della Droga'. L'UNODC ha un mandato per l'azione di controllo del fenomeno della Droga, cominciato nel I99I, ed un mandato per l'azione di prevenzione del Crimine, cominciato nel i997. In relazione a quest'ultimo l'UNODC è responsabile di prevenire il crimine, di contribuire all'attuazione della giustizia penale e di promuovere le riforme delle legislazioni. Nel campo della prevenzione del Crimine Internazionale, l'UNODC lavora insieme agli Stati Membri per rafforzare lo Stato di Diritto e creare degli stabili sistemi di giustizia penale; a tal fine promuove la firma, la ratifica e l'applicazione dei trattati internazionali, valuta le legislazioni nazionali esistenti e fornisce consulenza legale per

9 L'UNODC, originariamente United Nations International Drug Control Programme (UNDCP) istituito nel I99I, è stato rinominato tale nell'ottobre del 2002. Il suo staff è composto da circa cinquecento membri divisi tra gli Headquarters di Vienna, i ventidue Field Offices sparsi nel mondo, i quattro Regional Desks ed i due Liaison Offices di New York e Bruxelles. Il Programma è finanziato attraverso contributi volontari, provenienti dai Governi per il novanta per cento del budget complessivo. 
l'elaborazione, il rafforzamento e l'applicazione delle norme nazionali ed infine istruisce le forze giudiziarie e di polizia.

Esistono due corpi che affiancano l'UNODC nell'attività di prevenzione del Crimine: l'UNICRI e la Commission on Crime Prevention and Criminal Justice (CCPCJ) ${ }^{\text {Io }}$. Il primo è un Istituto di Ricerca che promuove studi sulle nuove ed emergenti figure di crimine; la seconda è stata creata nel I99I, come ente sussidiario del Consiglio Economico e Sociale. I quaranta membri della Commissione formulano le strategie internazionali e raccomandano le attività da svolgere nel campo del controllo sul crimine; è poi compito del Centre for International Crime Prevention portare avanti le decisioni della Commissione. La Commissione si riunisce annualmente a Vienna ed in occasione di tale sessione vengono elaborate delle Draft Resolutions, da sottoporre al Consiglio Economico e Sociale.

Se analizziamo la struttura dell'UNODC, possiamo notare che l'Anti-Trafficking Section fa parte dell'Human Security Branch della Division for Operations, cioè di quel settore dell'Ufficio incaricato di portare avanti i progetti di assistenza tecnica (operations) che sono finanziati dai Governi. Alla suddetta sezione appartiene, oltre all'Anti-Human Trafficking Unit, l'Anti-Organized Crime and Law Enforcement Unit ${ }^{\text {II }}$. L'Anti-Human Trafficking Unit è chiamata a fornire assistenza tecnica agli Stati membri per i progetti riguardanti la tratta d'esseri umani e a collaborare con i Field Offices e le altre unità competenti presso gli headquarters per lo sviluppo e l'implementazione del GPAT; è poi incaricata di formulare e diffondere le best practices, di analizzare i dati e le tendenze registrati sul fenomeno, di diffondere la coscienza sul problema della tratta, di istruire i professionisti chiamati ad operare nel campo della giustizia penale e gli addetti ai servizi di supporto alle vittime.

\section{Il sistema normativo di riferimento per l'azione delle Nazioni Unite.}

I. Definizione internazionalmente riconosciuta della fattispecie di tratta

La Comunità internazionale ha ormai da tempo preso coscienza del fatto che la tratta di persone, nella forma in cui si manifesta al giorno d'oggi, necessita di normative apposite, capaci di garantire la punibilità dei trafficanti anche nei casi in cui non sia possibile dimostrare l'intento di sottoporre la vittima all'esercizio di poteri inerenti il diritto di proprietà, così come richiesto dalla originaria nozione di tratta di schiavi. L'ostacolo maggiore ad un'efficace repressione del fenomeno consisteva, fino a poco tempo fa, nell'elaborazione, sul piano tanto internazionale che interno, di una definizione di tratta di persone sufficientemente determinata da rispondere ai canoni del principio di legalità della normativa penale ed, al contempo, sufficientemente ampia da comprendere tutte le diverse manifestazioni di un fenomeno in continua evoluzione quanto alle forme ed agli scopi.

Le numerose norme pattizie che si sono susseguite su questo tema, insieme ad altri elementi della prassi degli Stati, costituiscono adeguata prova dell'avvenuta formazione di una norma consuetudinaria che vieta la tratta mediante mezzi coercitivi di donne e minori a scopo di sfruttamento sessuale, matrimonio forzato o lavoro forzato ${ }^{\mathrm{r} 2}$. La necessità

\footnotetext{
Io Per maggiori informazioni sulla Commission on Crime Prevention and Criminal Justice si veda <http://www.unodc.org/unodc/en/commissions/CCPCJ/index.html> [consultato: 03-I2-2007].

II La struttura dell'UNODC è visibile presso <http://www.unodc.org/pdf/unodc_chart.pdf> [consultato: 03-I2-2007].

${ }^{12}$ Per una ricostruzione del processo di evoluzione del diritto internazionale nella lotta contro la tratta di esseri umani si veda DE ZulueTA, Relazione sul traffico di esseri umani, Commissione Parlamentare d'inchiesta sul fenomeno della mafia e delle altre associazioni criminali similari, XIII legislatura, doc. XXIII, n. 49, 2000, pp. 86 e ss.
} 
di conferire certezza a tale norma consuetudinaria, puntualizzando gli elementi costitutivi del crimine di tratta, ma anche la volontà di ampliare il contenuto del divieto, facendovi rientrare le condotte perpetrate ai danni dei maschi adulti e quelle finalizzate a nuove forme di sfruttamento della vittima, hanno spinto le Nazioni Unite all'elaborazione del Protocollo di Palermo del 2000 che prevede l'obbligo per gli Stati Parti di criminalizzare la tratta di qualsiasi essere umano realizzata a scopo di sfruttamento della vittima ${ }^{\mathrm{I3}}$.

Il peso e l'universalità della portata innovativa della nuova definizione di tratta di persone consacrano il successo di tale strumento. Prima del Protocollo, non solo non esisteva una norma che definisse la tratta di persone (trafficking) ${ }^{\mathrm{I}}$, ma esso veniva, in molti casi, confuso con la fattispecie del traffico di migranti (smuggling) ${ }^{\text {I5 }}$. Entrambi i fenomeni, infatti, comportano uno spostamento di persone da un luogo ad un altro, allo scopo di ottenere un profitto illecito. La differenza più evidente sta nel fatto che, in caso di trafficking, i trafficanti ottengono il controllo sulla vittima (ed anche l'eventuale consenso iniziale) attraverso mezzi di coercizione, inganno o abuso, mentre in caso di smuggling non può parlarsi di vittime del reato perché i migranti prestano il proprio consenso volontariamente, per quanto si tratti di persone che versano in condizioni di degrado o di pericolo. In secondo luogo, lo sfruttamento preventivato della vittima, una volta giunta nel luogo di destinazione, è un requisito essenziale nella fattispecie di tratta, mentre normalmente lo smuggling si conclude con l'arrivo a destinazione del migrante e l'eventuale successivo sfruttamento è considerato come aggravante nel Protocollo sullo smuggling. Inoltre l'illecito profitto ottenuto dai gruppi criminali organizzati, nel trafficking deriva dallo sfruttamento della vittima, mentre nello smuggling rappresenta il compenso pagato dai migranti per il trasporto. Ed infine, mentre la tratta va perseguita come reato sia che la vittima venga

${ }^{13}$ La Convenzione sul Crimine Transnazionale Organizzato e gli annessi Protocolli sulla Tratta di esseri umani e sul Traffico di Migranti, adottati dall'Assemblea Generale delle Nazioni Unite con la risoluzione 55/25 del I5 novembre 2000, rappresentano la risposta più significativa degli Stati, a livello internazionale, all'esigenza di fornire degli strumenti nuovi e più efficaci in grado di contrastare il dilagante fenomeno di una criminalità organizzata che ormai supera $\mathrm{i}$ confini nazionali, soprattutto nelle forme della tratta di persone e del traffico di migranti.

La Convenzione ed i Protocolli furono aperti alla firma da parte degli Stati durante la Conferenza Internazionale tenutasi a Palermo tra il I2 ed il I5 dicembre del 2000. I trattati raggiunsero un numero di firme senza precedenti (I23 per la Convenzione, II7 per il Protocollo sul trafficking e II2 per il Protocollo sullo smuggling). La Convenzione è entrata in vigore il 29 settembre del 2003 , mentre il Protocollo sul trafficking e quello sullo smuggling sono entrati in vigore rispettivamente il 25 dicembre 2003 ed il 28 gennaio 2004. Ad oggi i Paesi che hanno firmato la Convenzione sono I47 e quelli che l'hanno ratificata sono I38; i Paesi che hanno firmato il Protocollo sul trafficking sono II7 e quelli che hanno firmato quello sullo smuggling sono II2 mentre quelli che li hanno ratificati sono rispettivamente II6 e Io8 (cfr.: <http://www.unodc.org/unodc/en/treaties/CTOC/signatures.html>, consultato: 03-12-2007).

I4 La definizione internazionalmente riconosciuta della fattispecie di tratta di esseri umani è contenuta nell'articolo 3 lettera (a) del Protocollo sulla Prevenzione, Repressione e Punizione della Tratta di Persone, soprattutto donne e bambini, che recita: «Tratta di persone è il reclutamento, trasporto, trasferimento, sbarco o ricezione di persone, operati attraverso i mezzi della minaccia o dell'uso della forza o di altre forme di costrizione, rapimento, frode, raggiro, abuso di potere o di una posizione di vulnerabilità o attraverso l'offerta o la ricezione di denaro o altri vantaggi, con lo scopo che una persona ottenga il controllo su un'altra a fini di sfruttamento. Lo sfruttamento include, almeno, lo sfruttamento della prostituzione di altri o altre forme di sfruttamento sessuale, lavoro o servizi forzati, schiavitù o pratiche simili, asportazione di organi». L'articolo sopra citato descrive la natura del crimine indicando i tre elementi base la cui combinazione è richiesta per integrare la fattispecie di reato: innanzitutto gli atti nei quali si concreta la condotta criminosa, poi i mezzi utilizzati per il compimento di detti atti ed infine gli scopi prefissati di chi pone in essere tali atti.

${ }^{15}$ L'articolo 3 del Protocollo sul Traffico di Migranti per terra, mare ed aria definisce il fenomeno come segue: «Il traffico di migranti consiste nel procurare l'entrata illegale di una persona in uno Stato Parte di cui la persona non sia cittadino o residente permanente, al fine di ottenere, direttamente o indirettamente, un beneficio economico o altro beneficio materiale». 
condotta in altro Stato sia che venga spostata da un luogo ad un altro all'interno dei confini di uno Stato, lo smuggling prevede sempre come requisito necessario l'elemento della transnazionalità , trattandosi dell'entrata illegale in un altro Stato ${ }^{16}$.

Pur avendo presenti queste importanti distinzioni, può tuttavia dirsi che nella pratica può risultare difficile inquadrare una fattispecie sotto l'una o l'altra definizione. La tratta, infatti, spesso include lo smuggling o per meglio dire è uno smuggling a cui si aggiungono gli elementi di coercizione o inganno all'inizio del processo e di sfruttamento alla fine dello stesso. Conseguentemente, durante l'investigazione e la persecuzione del reato è, a volte, necessario utilizzare le misure previste contro lo smuggling fino al momento in cui gli investigatori o i difensori legali non scoprono gli ulteriori elementi tipici del trafficking, e per questo è importante che coloro che sono chiamati ad applicare la legge abbiano familiarità con entrambi i concetti ed entrambi i Protocolli. Inoltre la necessità di distinzione tra i due fenomeni nasce da ragioni politiche e da ragioni tecniche connesse con l'attività pratica delle forze chiamate a compiti di law enforcement ${ }^{17}$. Infatti a livello di competenze nazionali, le due attività sono spesso affidate ad organismi diversi ${ }^{18}$. D'altra parte, i due fenomeni hanno una rilevanza politica diversa: lo smuggling rappresenta una priorità nella gerarchia degli obiettivi politici di ogni singolo Stato, in quanto concerne problemi di sicurezza ed ordine pubblico, mentre la natura transnazionale del reato di tratta di persone richiede uno sforzo congiunto a livello internazionale per la prevenzione e la repressione del fenomeno ${ }^{\text {19 }}$.

\footnotetext{
${ }^{16}$ L'articolo 4 del Protocollo sul trafficking ne definisce i limiti di applicazione, stabilendo che esso verrà applicato per la prevenzione, inchiesta e persecuzione della tratta di persone, «qualora i reati abbiano natura transnazionale e coinvolgano un gruppo criminale organizzato». Ciò sembrerebbe escludere la possibilità di punire la tratta di persone perpetrata all'interno dei confini nazionali di uno Stato e quella compiuta da uno o due trafficanti. Questa ipotesi, che darebbe luogo ad un'ingiustificata disparità di trattamento tra due fattispecie entrambe di tratta, è tuttavia da escludere, in base a quanto risulta dalla Convenzione stessa all'articolo 34.2 che chiarisce che determinati reati, fra cui quelli previsti dall'articolo 5 della Convenzione, «devono essere previsti dalla legislazione nazionale di ogni Stato parte della Convenzione a prescindere dal carattere transnazionale e dal coinvolgimento di un gruppo criminale organizzato». Questa interpretazione trova espressa conferma nelle Legislative Guides pubblicate dall'UNODC ove si legge al paragrafo 25 che «in caso di tratta di persone, le norme penali nazionali dovranno essere applicate anche in assenza di transnazionalità del reato e di coinvolgimento di un gruppo criminale organizzato» (UNODC, Legislative Guides for the United Nations Convention against Transnational Organized Crime and the Protocols thereto, New York, 2004). Nella pratica giuridica, al fine di risolvere eventuali conflitti tra l'articolo 4 del Protocollo e l'articolo 34.2 della Convenzione, è bene chiarire che i difensori legali delle vittime non sono chiamati a provare i requisiti sopra citati per ottenere una condanna per tratta di persone o altro reato previsto dal Protocollo o dalla Convenzione, se si tratta di reati commessi e perseguiti all'interno di un singolo Stato ed al contrario devono provare l'esistenza di tali prerequisiti ove facciano richiesta di mutual legal assistance o di altra forma di cooperazione internazionale ad un altro Stato. Dunque la transnazionalità ed il coinvolgimento di un gruppo criminale organizzato non sono richiesti come elementi integranti la fattispecie di reato in sede di persecuzione nazionale.

${ }^{17}$ Cfr.: Savona, E.U., Belli, R., Curtol, F., DeCarli, S., De Nicola, A., Tratta di persone a scopo di sfruttamento e traffico di migranti, Roma: Transcrime, 2004, pp. 3I e ss.

${ }^{18}$ In Italia, ad esempio, la Direzione Centrale della Polizia Criminale presso il Dipartimento di Pubblica Sicurezza del Ministero dell'Interno ha competenza per i casi di trafficking, mentre il Servizio Stranieri dello stesso Dipartimento ha competenza per lo smuggling.

${ }^{19}$ In tal senso si pronuncia PÉREZ CEPEDA, A.I., «Instrumentos Internacionales en la lucha contra el trafico de inmigrantes y la trata de seres humanos», in Suplemento Boletin Europeo de la Universidad de La Rioja Io, julio 2002 , p. 46.
} 


\section{Armonizzazione delle legislazioni}

La cooperazione internazionale deve essere variamente indirizzata, ma non sussistono dubbi circa la principale necessità di fornire agli Stati assistenza tecnica per l'adeguamento delle legislazioni nazionali agli standards ed agli strumenti internazionali esistenti in materia e per la diffusione di una cooperazione giudiziaria in materia. A livello internazionale è importantissimo, dunque, che tutti gli Stati compiano il primo e cruciale passo di ratificare la Convenzione sul Crimine Transnazionale Organizzato ed i Protocolli ad essa annessi. A tal fine l'UNODC ha elaborato le Legislative Guides, che hanno lo scopo specifico di assistere gli Stati nel processo di ratificazione e/o attuazione della Convenzione e dei Protocolli ${ }^{20}$. Le Legislative Guides sono il risultato di un ampio processo di elaborazione cui hanno preso parte esperti e rappresentanti di Istituzioni e Governi di tutte le regioni del mondo. Esse sono state preparate per adattarsi a differenti tradizioni giuridiche ed ai diversi possibili livelli di sviluppo delle Istituzioni esistenti nei vari Paesi. Offrono, perciò, più opzioni d'attuazione, poiché i passi necessari per portare la legislazione nazionale in accordo con i nuovi strumenti internazionali possono risultare piuttosto complessi, dipendendo dallo stato di partenza della legislazione di ciascun Paese. Dato che le Guides sono essenzialmente destinate ai compilatori delle legislazioni nazionali, non tutte le previsioni della Convenzione e dei Protocolli sono in esse contenute e commentate, ma solo quelle che richiedono un cambio della normativa e/o quelle che richiedono il compimento di un'azione preventiva al fine di rendere applicabili, allo Stato in questione, gli strumenti internazionali.

Tra le previsioni che comportano delle obbligazioni di adeguamento normativo per gli Stati, con riguardo al Protocollo sulla Tratta, bisogna innanzitutto porre in rilievo l'importanza dell'articolo 5, che impone agli Stati la criminalizzazione di tutte le condotte comprese nella definizione di tratta fornita dall'articolo 3 del Protocollo stesso, qualora l'autore abbia agito «intenzionalmente» (cioè con dolo) ${ }^{2 \mathrm{t}}$. Inoltre si richiede che vengano parimenti puniti il tentativo di reato, la partecipazione in qualità di complice e l'organizzazione o direzione di altre persone per la commissione del reato. A proposito del tentativo, è stabilito che esso venga punito «in accordo con i concetti base di ciascun sistema legale». Tale locuzione si è resa necessaria poiché il concetto di tentativo non esiste nei sistemi legislativi penali di alcuni Paesi e dunque la previsione, pur avendo una portata generalmente obbligatoria, non può essere considerata «fully mandatory» nei Paesi in cui ciò risulti effettivamente impossibile. In secondo luogo, molti Stati, anche se non è richiesto dal Protocollo, hanno criminalizzato una serie di condotte relazionate con la tratta di persone. Bisogna considerare, infatti, che la tratta è solo uno dei crimini commessi contro le persone trafficate e che altri crimini sono compiuti per assicurarsi l'obbedienza della vittima e mantenere il controllo su di essa, per occultare le operazioni di tratta o per ottimizzare i profitti. Tutti questi atti costituiscono reati in molti Stati e ciò può rivelarsi molto utile laddove il reato specifico di tratta ancora non esiste o laddove le pene per tale reato sono insufficienti. Possono inoltre esservi casi in cui la prova di cui si dispone non è idonea a supportare la persecuzione per il reato di tratta, ma lo è invece ai fini della persecuzione di reati ad essa correlati. Se poi tali reati sono puniti, sotto la legge nazionale,

${ }^{20}$ UNODC, Legislative Guides for the United Nations Convention against Transnational Organized Crime and the Protocols thereto, United Nations, New York, 2004.

${ }^{21}$ Articolo 5: «(I) Ogni Stato Parte adotta le misure legislative e di altro tipo necessarie per conferire il carattere di reato alla condotta di cui all'articolo 3 del presente Protocollo,quando posta in essere intenzionalmente. (2) Ogni Stato Parte adotta le misure legislative e di altro tipo necessarie per conferire il carattere di reato: a) fatti salvi i concetti fondamentali del suo ordinamento giuridico, al tentativo di commettere un reato determinato ai sensi del paragrafo I del presente articolo; b) alla partecipazione, in qualità di complice, ad un reato determinato ai sensi del paragrafo I del presente articolo; c) all'organizzare o dirigere altre persone nella commissione di un reato determinato ai sensi del paragrafo I del presente articolo». 
con almeno quattro anni di reclusione, essi sono qualificabili come «serious crimes» ai sensi della Convenzione, che risulterà, dunque, direttamente applicabile ad essi.

Il reato di tratta e quelli ad esso associati sono spesso commessi attraverso o sotto la copertura di enti giuridici, come società o false organizzazioni a scopo benefico. Per questa ragione, l'articolo io della Convenzione richiede agli Stati l'adozione di misure adeguate che prevedano la responsabilità delle persone giuridiche per il caso di partecipazione alla commissione di un «serious crime» da parte di un gruppo criminale organizzato $^{22}$. Tale responsabilità può essere penale, civile o amministrativa, nel rispetto della posizione dei diversi sistemi giuridici sul tema della responsabilità delle persone giuridiche. Essa non deve pregiudicare, inoltre, l'eventuale responsabilità penale delle persone fisiche che hanno commesso il reato; allorché un individuo commette un crimine per conto di una persona giuridica, deve essere possibile agire contro di entrambi. Gli Stati sono chiamati, infine, ad assicurare che la persona giuridica considerata responsabile sia sottoposta ad effettive e proporzionate sanzioni di natura penale o meno, incluse quelle monetarie.

Una buona strategia nazionale per combattere il traffico di esseri umani deve includere, inoltre, un efficace regime di confisca che preveda l'identificazione, il congelamento e la confisca legale dei fondi e delle proprietà illecitamente acquisiti. La criminalizzazione del riciclaggio dei proventi dei reati connessi alla tratta costituisce parte importante di una strategia globale. L'articolo 6 della Convenzione richiede che gli Stati prevedano i quattro seguenti reati relazionati con il riciclaggio del denaro: conversione $o$ trasferimento dei proventi del reato con lo scopo di occultarne l'origine illecita; occultamento o contraffazione dei proventi del reato; acquisizione, possesso o utilizzo dei proventi del reato; contribuzione, anche indiretta, alla commissione dei reati sopra enunciati, nelle forme della partecipazione, della cospirazione o del tentativo ${ }^{23}$.

${ }^{22}$ Articolo IO: «(I) Ogni Stato Parte adotta misure necessarie, conformemente ai suoi princìpi giuridici, per determinare la responsabilità delle persone giuridiche che partecipano a reati gravi che coinvolgono un gruppo criminale organizzato e per i reati di cui agli articoli 5, 6, 8 e 23 della presente Convenzione. (2) Fatti salvi i princìpi giuridici dello Stato Parte, la responsabilità delle persone giuridiche può essere penale, civile o amministrativa. (3) Tale responsabilità è senza pregiudizio per la responsabilità penale delle persone fisiche che hanno commesso i reati. (4) Ogni Stato Parte si assicura, in particolare, che le persone giuridiche ritenute responsabili ai sensi del presente articolo siano soggette a sanzioni efficaci, proporzionate e dissuasive, di natura penale o non penale, comprese sanzioni pecuniarie».

${ }^{23}$ Articolo 6 della Convenzione: «(I) Ogni Stato Parte adotta, conformemente ai princìpi fondamentali della sua legislazione interna, le misure legislative e di altra natura, necessarie a conferire il carattere di reato, laddove commessi intenzionalmente: alla conversione $\mathrm{o}$ al trasferimento di beni, sapendo che tali beni costituiscono proventi di reato, al fine di occultare o dissimulare la provenienza illecita dei beni o di aiutare qualsiasi persona coinvolta nella commissione del reato presupposto ad eludere le conseguenze giuridiche della sua azione; all'occultamento o alla dissimulazione della vera natura, fonte, ubicazione,cessione, movimento o proprietà di beni o di diritti su questi beni, sapendo che tali beni sono provento di reato; fatti salvi i concetti fondamentali del suo ordinamento giuridico: all'acquisizione, possesso o utilizzo dei beni, sapendo, al momento in cui li riceve, che tali beni sono il provento di reato; alla partecipazione, associazione, accordo, tentativo per commettere, facilitare, incoraggiare, favorire o consigliare, finalizzati alla commissione di qualunque dei reati di cui al presente articolo. (2) Ai fini dell'attuazione o applicazione del paragrafo I del presente articolo: a) ogni Stato Parte cerca di applicare il paragrafo I del presente articolo alla più vasta gamma possibile di reati presupposti; b) ogni Stato Parte include nella categoria di reati presupposti tutti i reati gravi come definiti all'articolo 2 della presente Convenzione e i reati determinati conformemente agli articoli 5, 8 e 23 della presente Convenzione. Nel caso di Stati Parte la cui legislazione contiene una elencazione di reati presupposti specifici, essi includono, per lo meno, una gamma completa di reati connessi a gruppi criminali organizzati; c) ai fini della lettera b), i reati presupposti comprendono reati commessi sia all'interno che all'esterno della giurisdizione dello Stato Parte in questione. Tuttavia, i reati 
A livello interno, d'altra parte, si richiede un'ampia ed approfondita valutazione del sistema normativo di ciascun Paese con riguardo alla fattispecie di tratta; essa deve comprendere l'analisi delle leggi penali, di diritto del lavoro, sull'immigrazione e sul diritto d'asilo, sui servizi sociali, sul crimine organizzato, sulla prostituzione, nonché l'analisi delle procedure investigative e giudiziarie ${ }^{24}$.

\section{Sviluppo di una strategia a livello nazionale}

Una risposta efficace al problema del traffico di esseri umani esige un'azione ben pianificata ed a lungo termine nonché una collaborazione coordinata tra le diverse forze chiamate a collaborare. La pianificazione dell'azione deve essere basata su una valutazione complessiva del problema e delle capacità di cui si dispone per affrontarlo e deve essere supportata dalla disponibilità dei vari gruppi e delle agenzie locali che sono chiamate a cooperare tra loro e con altre agenzie a livello internazionale. Data la complessità del fenomeno, infatti, è piuttosto improbabile raggiungere un qualche successo a livello nazionale senza una collaborazione tra le agenzie, al fine di delineare un Piano d'Azione Nazionale, con obiettivi predefiniti, priorità d'intervento, risorse richieste e relativa responsabilità di ciascun operatore.

Forniamo, qui di seguito, alcuni esempi elaborati al di fuori del sistema delle Nazioni Unite. Il Consiglio Permanente dell'Organization for Security and Cooperation in Europe (OSCE), per esempio, con la decisione numero 557 del 24 luglio 2003, ha adottato l'Action Plan to Combat Trafficking in Human Beings: esso affronta il problema in maniera onnicomprensiva, preoccupandosi della protezione delle vittime, della prevenzione del fenomeno e della persecuzione contro coloro che hanno commesso o facilitato il crimine ${ }^{25}$. L'Office for Democratic Institutions and Human Rights dell'OSCE ha sviluppato, inoltre, un Practical Handbook, National Referral Mechanism - Joining Efforts to Protect the Rights of Trafficked Persons, che contiene tre utili strumenti da utilizzare durante il processo di valutazione del fenomeno, ciascuno dei quali concentra l'attenzione su un differente oggetto: il primo sulle condizioni ed i bisogni specifici del Paese, il secondo sul sistema

commessi al di fuori della giurisdizione di uno Stato Parte costituiscono reati presupposti soltanto quando la relativa condotta costituisce reato ai sensi del diritto interno dello Stato dove è commesso e sarebbe stato reato ai sensi del diritto interno dello Stato Parte che attua o applica il presente articolo se il reato fosse stato ivi commesso; d) ogni Stato Parte fornisce copia delle sue leggi che danno efficacia al presente articolo e delle successive modifiche a tali leggi o una descrizione di esse al Segretario Generale delle Nazioni Unite; e) se richiesto dai princìpi fondamentali del diritto interno di uno Stato Parte, può essere disposto che i reati di cui al paragrafo I del presente articolo non si applichino alle persone che hanno commesso il reato presupposto; f) la conoscenza, l'intenzione o lo scopo, in quanto elementi di un reato di cui al paragrafo I del presente articolo, possono essere dedotti da circostanze obiettive basate su fatti».

${ }^{24}$ A titolo di esempio, in Vietnam il Department of Criminal and Administrative Laws del Ministero della Giustizia, in collaborazione con l'UNODC e l'UNICEF, ha realizzato un progetto di valutazione del sistema legislativo esistente in comparazione con i requisiti richiesti dalla Convenzione e dai Protocolli sulla tratta e sul traffico di migranti.

Cfr.: <www.unodc.org/pdf/crime/human_trafficking/legal_system_vietnam_2004-o5.pdf> [consultato: 03-I2-2007].

${ }^{25}$ OSCE Permanent Council Decision N. 57, Action Plan to Combat Trafficking in Human Beings, 23 July 2003, <http://www.osce.org/documents/pc/2003/07/724_en.pdf> [consultato: 03-I2-2007]. 
normativo esistente ed il terzo sugli attori, vale a dire, sulle organizzazioni che si occupano di contrastare il fenomeno ${ }^{26}$.

La valutazione di un progetto portato avanti nelle Filippine dall'UNODC e dall'UNICRI ha palesato una situazione che è in realtà tipica di molti Stati. Al tempo dell'apprezzamento, un numero consistente d'agenzie e dipartimenti governativi si stava già occupando dei vari aspetti dei fenomeni di trafficking e di smuggling, ciascuno secondo le rispettive aree di competenza. Nonostante tali enti stessero lavorando per raggiungere gli stessi obiettivi, essi spesso operavano in maniera indipendente, inconsapevoli dell'azione degli altri enti. Il risultato è stato quello di una duplicazione di sforzi e di una conseguente inefficienza. Il rapporto del progetto in questione ha rilevato, quindi, la necessità di rafforzare la cooperazione tra le agenzie governative ed il sistema di giustizia penale. In particolare, ha posto l'accento sul debole grado di coordinamento tra la polizia ed i magistrati dell'accusa, fattore che ha certamente condotto all'erronea chiusura di più casi giudiziari $^{27}$.

Una volta valutato il fenomeno nella sua dimensione nazionale, si passa all'elaborazione della strategia per sconfiggerlo. Il contenuto di ciascun Piano d'Azione è ovviamente determinato sulla base delle circostanze nazionali, ma ciò non toglie che vi siano spesso dei punti comuni tra i diversi Piani d'Azione, quali la programmazione degli obiettivi da raggiungere e delle priorità d'intervento e l'identificazione, per ogni fase, dei responsabili della realizzazione e delle rispettive risorse richieste. I Piani più completi prevedono anche una fase di monitoraggio e di valutazione finale del raggiungimento degli obiettivi prefissati.

Successivamente all'adozione del Piano d'Azione, occorre, prima di tutto, comunicarlo a quanti sono interessati alla sua messa in atto al fine di creare un'ampia rete di supporto dello stesso; in secondo luogo, procurare le risorse umane e finanziarie necessarie per la sua realizzazione ed infine stabilire dei tempi limite e degli standards per l'attuazione ed il monitoraggio. In aggiunta, sviluppare un approccio che coinvolga diverse agenzie e dei meccanismi di coordinamento per l'azione di queste, nonché rafforzare la capacità istituzionale di mettere in atto un'ampia strategia d'intervento sono punti vincenti della sfida. Considerando la natura del fenomeno di tratta ed i reati connessi, da un lato, e le molteplici necessità delle vittime, dall'altro, risulta chiaro come l'esperienza richiesta non possa provenire da un'unica fonte; un sistema di organizzazione e direzione delle operazioni va dunque concordato tra i diversi attori, magari sulla base dei patti già esistenti. Un sistema del genere comporta: identificare contatti-chiave ed agenzie di rappresentanza, stabilire contatti personali tra gli operatori delle diverse agenzie, realizzare un training comune alle diverse agenzie, mettere in comune intelligence e dati, elaborare Protocolli per un'azione congiunta e assicurare una forte ed efficiente leadership dei diversi partner.

A proposito di strategie risolutive, è suggerita come best practice l'adozione di protocolli formali tra la polizia e le organizzazioni non governative locali, al fine di stabilire i compiti e le responsabilità dei due lati e di dirigere lo scambio di dati ed informazioni riservate. A titolo di mero esempio, un protocollo potrebbe decidere che spetta agli investigatori occuparsi della formazione e conservazione della prova e garantire la presenza

\footnotetext{
${ }^{26}$ OSCE/ODIHR, National Referral Mechanism: Joining the Efforts to Protect the Rights of Trafficked Persons, 2004, <http://www.osce.org/documents/odihr/2004/05/2903_en.pdf> [consultato: 03-I22007].

${ }^{27}$ Cfr.: UNICRI/AIC, Rapid Assessment: Human Smuggling and Trafficking from the Philippines, November I999, <www.unodc.org/pdf/crime/trafficking/RA_UNICRI.pdf> [consultato: 03-I22007].

Una valutazione più completa della situazione nelle Filippine è stata condotta nell'ambito del progetto UNODC/UNICRI «Coalitions Against Trafficking in Human Beings», 2003, <www.unodc.org/pdf/crime/human_trafficking/coalitions_trafficking.pdf> [consultato: 03-I2-2007].
} 
della vittima per l'identificazione e nelle comparizioni in tribunale, mentre spetta all'agenzia che si occupa del supporto alle vittime fornire la presenza di un avvocato in caso d'interrogatorio o comparizione della vittima in tribunale ed avanzare richiesta per lo status temporaneo di residente o altri benefici del genere.

I meccanismi di coordinamento d'azione tra le diverse agenzie hanno lo scopo di mettere in atto le strategie comuni, monitorare la loro realizzazione, coordinare l'operato dei diversi attori a livello nazionale e facilitare la cooperazione internazionale. Un meccanismo è di successo se definisce in maniera chiara e precisa i ruoli delle diverse agenzie chiamate all'azione (who does what locally) e se su tali ruoli è stato raggiunto un $\operatorname{accordo}^{28}$.

\section{Cooperazione internazionale nel campo della giustizia penale}

I migliori risultati nella persecuzione di un reato si ottengono laddove le forze di polizia ed i magistrati dell'accusa sono capaci di lavorare bene insieme, sia a livello locale, sia oltre i confini nazionali. Se è vero, infatti, che accordi ad hoc, trattati sulla reciproca assistenza legale e sull'estradizione possono garantire risultati soddisfacenti in alcuni casi, è anche vero d'altra parte che la complessità dei sistemi legislativi e procedurali dei diversi Paesi ha impedito a tale tipo di strumenti di essere sufficientemente efficaci in ogni situazione. Da qui è nata l'esigenza di nuove e specifiche Convenzioni sul traffico di droga, sul terrorismo, sulla corruzione, sul riciclaggio di denaro, etc. La Convenzione sul Crimine Transnazionale Organizzato prevede un sistema di cooperazione internazionale per combattere il crimine organizzato, ragion per cui l'adesione a tale strumento rimuove la maggior parte degli ostacoli che, prima di esso, hanno impedito agli Stati di collaborare in tal senso.

\section{Estradizione}

I responsabili di un illecito transnazionale possono vivere in uno Stato diverso da quello in cui è stato consumato il reato oppure fuggire in un altro Stato con lo scopo di evitare la persecuzione. In tal caso diventa necessario un procedimento d'estradizione per condurre il colpevole davanti al tribunale dello Stato procedente. L'estradizione è un procedimento formale, basato, nella maggioranza dei casi, su un trattato; sin dal diciannovesimo secolo gli Stati hanno firmato accordi di questo tipo ed essi variano da Stato a Stato, coprendo differenti fattispecie di reati. Con l'avanzare degli anni, per evitare l'inconveniente di aggiornare con frequenza la lista dei trattati al fine di includere nuovi

${ }^{28}$ Forniamo un esempio tratto dall'esperienza governativa statunitense. Il Trafficking Victims Protection Act del 2000 ha richiesto la creazione della President's Interagency Task Force to Monitor and Combat Trafficking in Persons per coordinare gli sforzi tra le diverse agenzie governative federali degli Stati Uniti d'America. Il Department of Health and Human Services è designato quale agenzia responsabile di certificare il numero delle vittime di tratta dopo la loro identificazione, al fine di permettere a queste l'accesso ai benefici ed ai servizi (alle stesse condizioni dei rifugiati) finanziati a livello federale. Il Department of Justice ha il compito di investigare sui casi di tratta e di promuovere azione penale contro i trafficanti; inoltre ha creato un sistema che facilita il reclamo a quanti vogliono denunciare un caso di tratta. Il Department of Labor offre alle vittime programmi di assistenza per la ricerca di lavoro, servizi di consulenza sul lavoro, servizi di educazione e formazione, informazioni sui servizi di trasporto, sugli alloggi ed altro. Il Department of State è responsabile del coordinamento dei programmi internazionali contro il traffico di esseri umani. Infine il Department of Homeland Security comprende il U.S. Citizenship and Immigration Services ed il Customs Enforcement. Quest'ultimo fornisce supporto nell'investigazione dei casi di tratta e per l'identificazione delle vittime, mentre il primo concede il visto speciale ( $T$ visa) e lo status conseguente che permettono alla vittima di essere certificata. 
reati, emersi in seguito al progresso della tecnologia ed ai cambiamenti socio-economici, si è deciso di fare ricorso al principio della «doppia incriminazione», che si applica allorché la stessa condotta sia prevista come reato sia nello Stato richiedente sia nello Stato cui è stata avanzata richiesta d'estradizione ed allorché le pene per esso previste non superino una determinata soglia. Inoltre, per far fronte all'esigenza di un approccio multilaterale, in diverse regioni del mondo sono state prese iniziative con la prospettiva di dare alla problematica una soluzione comune; tra queste si segnalano l'Economic Community of West African States Convention on Extradition del I994, cui si è aggiunto uno strumento più innovativo quale il Mandato d'Arresto Europeo, introdotto con il fine di semplificare le procedure d'estradizione ${ }^{29}$.

Per dare attuazione alle disposizioni sull'estradizione contenute nella Convenzione sul Crime Transnazionale Organizzato, gli Stati, dipendendo dalla misura in cui la legislazione nazionale ed i trattati preesistenti disciplinano l'argomento, possono aver bisogno di rivedere e correggere la propria legislazione o addirittura introdurre un nuovo sistema per l'estradizione. In tale processo d'adeguamento, i legislatori devono tener presente che lo scopo della Convenzione è quello di assicurare un giusto trattamento a coloro di cui si richiede l'estradizione, mediante l'applicazione di tutti i diritti e le garanzie previste secondo la giurisdizione del Paese richiedente. Gli Stati membri dei Protocolli sul trafficking e sullo smuggling devono, inoltre, assicurarsi che le proprie legislazioni consentano l'estradizione per i rispettivi reati.

Infine, è importante evidenziare che le Nazioni Unite hanno elaborato un Model Treaty sull'estradizione per assistere gli Stati interessati alla negoziazione ed alla conclusione di accordi bilaterali volti a migliorare la cooperazione in materia di prevenzione del crimine e giustizia penale ${ }^{30}$.

\section{Reciproca Assistenza Legale}

In un contesto globalizzato, le autorità nazionali necessitano dell'assistenza di altri Stati per portare a compimento con successo l'inchiesta, la persecuzione e la condanna dei colpevoli del reato di tratta di persone. La mobilità internazionale di questi ultimi e l'utilizzo di tecnologie sempre più avanzate rende tale bisogno sempre più frequente ed urgente. A tal fine gli Stati hanno provveduto con Trattati di Reciproca Assistenza Legale in materia penale. Queste intese bilaterali hanno la finalità di rafforzare il sistema d'applicazione della legge in diversi modi. In primo luogo, consentono alle autorità competenti di acquisire le prove all'estero in un modo tale da renderle ammissibili ai sensi della legge interna; in secondo luogo, fanno da supplemento ad altri accordi che regolano lo scambio d'informazioni (ottenute attraverso l'INTERPOL, i rapporti tra i diversi uffici di polizia, etc.) ed infine risolvono certe complicazioni che si creano tra Stati con differenti tradizioni giuridiche, giacché alcuni tra questi limitano la possibilità d'assistenza alle autorità giudiziarie.

La Convenzione sul Crimine Transnazionale Organizzato esige l'adozione di misure di reciproca assistenza legale quanto più ampie possibili durante la fase d'indagine e di persecuzione nonché durante il procedimento giudiziario. L'assistenza può essere

${ }^{29}$ Per maggiori approfondimenti sul Mandato d'Arresto Europeo si veda $<$ http://ec.europa.eu/justice_home/fsj/criminal/extradition/fsj_criminal_extradition_en.htm> [consultato: 03-I2-2007].

${ }^{30}$ Assemblea Generale Delle NaZioni Unite, Risoluzione 53/iin del i4 dicembre i990, «Model Treaty on Extradition», in UN Official Records of the General Assembly, 68th plenary meeting (A/RES/45/ir6). 
richiesta a diversi fini: per la formazione della prova, per ottenere dei documenti indispensabili per il giudizio, per eseguire ricerche e confische, per l'esame d'oggetti e luoghi, per valutazioni di periti, per rintracciare i proventi del reato, per facilitare la comparizione dei testimoni ed in ogni altro caso non vietato dalla legge nazionale. La Convenzione, riconoscendo la diversità dei sistemi giuridici, consente agli Stati di rifiutare una richiesta d'assistenza alla presenza di determinate condizioni indicate nell'articolo i8 della stessa, ma purché il rifiuto sia sempre adeguatamente motivato.

Una richiesta di reciproca assistenza legale può essere avanzata in tre distinte maniere: attraverso i canali diplomatici (quando non esistono dei precedenti accordi tra gli Stati in questione), attraverso i canali previsti ai sensi degli accordi bilaterali o attraverso quelli stabiliti da convenzioni multilaterali. Un problema diffuso è determinato dalla lentezza con cui tali richieste attraversano gli uffici legali ed amministrativi, compresi i servizi di traduzione. Altre problematiche derivano dalla scarsa qualità della forma in cui esse sono presentate, che non fa chiarezza sulle azioni richieste, determinando di conseguenza un ritardo nella risposta. L'UNODC ha creato un mutual legal assistance request writer tool per aiutare gli operatori non esperti ad ottimizzare la procedura; esso è pratico, facilmente adattabile alle leggi sostanziali e procedurali degli Stati e non richiede la previa conoscenza della materia da parte degli addetti al servizio. Il request writer tool guida l'operatore, passo dopo passo, nella procedura, attraverso delle schermate che contengono dei modelli di riferimento per ogni tipo di richiesta. Al fine di evitare il rischio di rifiuto o di ritardo, esso avverte l'operatore dell'eventuale mancanza di informazioni obbligatorie e, quando tutti i dati sono stati inseriti, li rielabora e fornisce il modulo di richiesta completo e corretto, pronto ad essere firmato ${ }^{31}$.

\section{Altre forme di cooperazione}

Una richiesta formale di reciproca assistenza legale non è l'unica via per ottenere collaborazione in materia giuridica da un altro Stato. Tale tipo di procedura è, in realtà, limitata ai soli casi in cui sono necessarie delle misure coercitive, dato che queste ultime normalmente richiedono l'intervento dell'autorità giudiziaria. Altre possibilità, meno formali, di cooperazione, possono essere messe in atto. La Convenzione sul Crimine Transnazionale Organizzato prevede una serie di meccanismi obbligatori e non obbligatori per facilitare la cooperazione internazionale, tra cui le operazioni congiunte d'indagine. Secondo l'articolo I9, uno Stato membro deve propendere per la conclusione d'accordi bilaterali o multilaterali che istituiscano corpi d'indagine congiunta, ma allo stesso tempo far sì che la sovranità dello Stato nel cui territorio l'indagine è portata avanti sia pienamente rispettata ${ }^{32}$.

Un rilevante esempio della capacità degli Stati di sviluppare una rete di contatti internazionali per migliorare la cooperazione nel campo del law enforcement, specie con riguardo alle attività del crimine organizzato, è rappresentato dall'istituzione di funzionari di contatto esperti della materia, secondo quanto suggerito nell'European Convention on Mutual Legal Assistance in Criminal Matters del 29 maggio del 2000. Il Consiglio

${ }^{31}$ UNODC, Mutual legal assistance request writer tool, <http://www.unodc.org/mla/> [consultato: 03-I2-2007].

${ }^{32}$ Articolo I9: «Gli Stati Parte valutano l'opportunità di stringere accordi o intese bilaterali o multilaterali per mezzo dei quali, rispetto a questioni oggetto di indagini, azioni penali o procedimenti giudiziari in uno o più Stati, le autorità competenti interessate possono creare organi investigativi comuni. In mancanza di tali accordi o intese, si possono intraprendere indagini comuni sulla base di accordi caso per caso. Gli Stati Parte coinvolti assicurano il pieno rispetto della sovranità dello Stato Parte nel cui territorio tale indagine ha luogo». 
dell'Unione Europea, ad esempio, ha istituito un corpo di magistrati esperti nella lotta contro la criminalità organizzata transnazionale, EUROJUST, con sede a l'Aja e composto da un magistrato per ogni Stato membro ${ }^{33}$. EUROJUST deve mantenere una stretta cooperazione con EUROPOL, collaborare e consultarsi con la Rete giudiziaria europea, creare un efficace sistema di scambio d'informazioni tra i Paesi. Altro esempio è quello della Southeast European Cooperative Initiative (SECI), che ha visto riunire gli sforzi di undici Paesi dell'area balcanica per la creazione di un centro di funzionari di polizia e di dogana, con sede a Bucarest, che facilita lo scambio d'informazioni tra le forze di polizia dei diversi Paesi.

Può essere di grande utilità, in proposito, combinare incontri internazionali tra gli ufficiali di polizia dei diversi Paesi con finalità d'aggiornamento e di scambio; a tal fine, ad esempio, l'INTERPOL ha creato un gruppo di lavoro sulla tratta di donne a scopo di sfruttamento sessuale, che è aperto agli agenti di polizia di tutti i I79 Stati membri. L'INTERPOL dispone, inoltre, di National Central Bureaus, che mantengono i contatti con i dipartimenti degli altri Stati. Attraverso questo tipo di collegamenti, possono essere scambiate delle importanti informazioni sull'identità e sulle attività dei soggetti coinvolti nel caso (sospettati, vittime o testimoni), sui numeri di telefono, sui veicoli, sugli indirizzi e sui documenti usati da tali soggetti; uno scambio del genere rappresenta, spesso, il primo passo che conduce ad una richiesta formale di reciproca assistenza legale.

Ancora con riguardo alla cooperazione tra le forze di polizia, la Convenzione sul Crimine Transnazionale Organizzato richiede agli Stati Parti di sviluppare tra loro una stretta collaborazione per potenziare l'effettiva attuazione della legge, attraverso lo scambio d'informazioni, l'identificazione dei colpevoli, la localizzazione delle vittime, dei testimoni, dei movimenti dei proventi e delle proprietà, nonché dei responsabili dei reati. Si può procedere ad un accordo di cooperazione internazionale che comporta assistenza operativa della polizia prima dell'instaurazione di un procedimento penale (ed in tal caso dovrà essere accertato chi ha l'autorità per autorizzare le attività in questione) o dopo (ed in tal caso la procedura è quella della presentazione di una lettera di richiesta). In molte giurisdizioni, inoltre, i contatti bilaterali o multilaterali diretti tra gli investigatori sono scoraggiati, o addirittura vietati, in nome di una volontà di centralizzazione dei controlli e di standardizzazione delle risposte. In caso d'urgenze, determinate da rischi vari, quali la sicurezza della vittima, la dispersione della prova, la fuga del sospettato, possono, dunque, essere stabiliti contatti solo attraverso l'INTERPOL, cui va dimostrata la sussistenza di un rischio specifico.

Infine la Convenzione incoraggia gli Stati alla conclusione di altri accordi bilaterali di cooperazione, nella forma, ad esempio, di Memorandum of Understanding ${ }^{34}$.

\section{Metodi d'indagine}

Le strategie investigative devono essere studiate prendendo in considerazione gli elementi geografici, strutturali e commerciali del reato di tratta d'esseri umani. Combinando le prime due categorie di elementi, possiamo elaborare il seguente schema:

33 Consiglio Dell'unione EuropeA, Decisione 2002/187/GAI del 28 febbraio 2002 che istituisce l'EUROJUST per rafforzare la lotta contro le forme gravi di criminalità, Gazzetta Ufficiale delle Comunità Europee <http://www.giustizia.it/misc/1_06320020306itoooıooI3.pdf> [consultato: 03I2-2007].

${ }^{34}$ Uno di questi è stato firmato tra Nigeria ed Italia al fine di rafforzare la capacità di prevenzione, indagine e persecuzione dei casi di tratta da parte delle forze di polizia nigeriana:

<http://www.unicri.it/wwd/trafficking/nigeria/memorandum.php> [consultato: 03-I2-2007]. 
- Stato d'origine: reclutamento delle vittime;

- Stato di transito: trasferimento delle vittime;

- Stato di destinazione: ricezione e sfruttamento delle vittime.

All'interno di tale divisione, s'inseriscono le caratteristiche commerciali del reato, che trovano espressione in diverse attività attinenti ad una o più delle fasi sopra citate:

- pubblicità (come parte del processo di reclutamento o di sfruttamento);

- affitto dei locali;

- trasporto mediante documenti d'identità e di viaggio;

- comunicazioni;

- transazioni finanziarie.

Gli investigatori devono trovare la via per reperire il materiale utile in termini probatori, che può emergere durante il processo di tratta. A tal fine esistono differenti approcci investigativi, i quali non si escludono l'uno con l'altro. Il metodo d'indagine «reattiva», vale a dire conseguente ad un reclamo da parte di una o più vittime, anche se non è il più efficace, diventa a volte necessario a causa delle immediate esigenze di protezione della vittima o delle prove da rischi attuali. L'intervento degli investigatori, nelle forme dell'arresto, è tempestivo nei confronti dei trafficanti, perché mira a soccorrere altre vittime o a prevenirne l'ulteriore reclutamento o ancora a rendere certa l'acquisizione di una prova, che andrebbe altrimenti perduta. Laddove il tempo lo consenta, appare più opportuno, prima di procedere all'arresto dei sospettati, fare una ricognizione delle premesse per valutare il numero delle persone presenti nel luogo, la presenza di porte blindate, il numero delle stanze. L'intervento reattivo spesso può rivelarsi inefficace, perché vittime che hanno inizialmente avanzato reclamo e promesso di testimoniare davanti al giudice, ritirano in un secondo momento la loro offerta di collaborazione.

Altro metodo è quello dell'indagine «proattiva», vale a dire che l'indagine, l'arresto e la persecuzione dei trafficanti avvengono senza la collaborazione e la testimonianza delle vittime. Tale scelta prende atto della realtà che le vittime potrebbero non volere o potere testimoniare contro i propri trafficanti e si basa dunque su altri mezzi, quali la sorveglianza, il dispiegamento di un sistema segreto ed i servizi d'intelligence. Esso si è rivelato molto efficace, perché parte da una considerazione di base destinata a rimanere valida nel tempo, e cioè quella secondo cui possono cambiare tutti gli elementi del reato meno uno: la necessità dei trafficanti di commercializzare il prodotto. Tale imperativo commerciale costituisce, appunto, il «tallone d'Achille», che gli investigatori possono sfruttare per localizzare prima le vittime e poi, di conseguenza, i trafficanti. Vi possono essere dei casi in cui entrambi i metodi sopra citati non sono praticabili; per tali evenienze, sono stati pensati dei metodi alternativi che hanno lo scopo di interrompere le operazioni di tratta. Ovviamente tale tipo di intervento si risolve semplicemente in un arresto temporaneo del fenomeno, che non conduce ad una soluzione, limitandosi a spostare il problema in altro luogo. La chiave di successo di queste operazioni risiede nell'utilizzo di tattiche creative ed innovative, che creano nel luogo così tanti problemi quotidiani, da rendere impossibile ai trafficanti continuare ad operare in quelle condizioni. Queste tattiche richiedono il coinvolgimento di molteplici enti: forze di polizia, servizi per l'immigrazione, Ministero della Salute, dell'Ambiente, del Lavoro, degli Affari Esteri, autorità comunali, aerolinee.

Durante la fase d'indagine, non bisogna sottovalutare il ruolo della componente finanziaria del reato. La regola d'oro è «follow the money and you will find the trafficker». Si dice comunemente, infatti, che «the crime itself is all about money», affermazione che non 
possiamo che confermare considerando che, a parte l'investimento iniziale necessario per creare l'infrastruttura, la gestione dei profitti derivanti dallo sfruttamento delle vittime ed il riciclaggio degli stessi sono ormai parte delle attività dei trafficanti. La fase d'indagine può trovare un decisivo contributo nella collaborazione di membri d'organizzazioni criminali coinvolti in tale tipo d'attività. L'articolo 26 della Convenzione sul Crimine Transnazionale Organizzato richiede agli Stati l'adozione, nei limiti del rispetto dei principi giuridici fondamentali, di misure per incoraggiare i soggetti che fanno o hanno fatto parte di un gruppo criminale organizzato a fornire informazioni per scopi d'indagine o probatori ed a contribuire concretamente con le forze di polizia per privare i gruppi criminali delle proprie risorse e dei profitti. D'altra parte richiede per tali soggetti la possibilità di previsioni di pene ridotte $\mathrm{o}$, in alcuni casi addirittura, d'immunità dalla persecuzione ${ }^{35}$.

\section{Assistenza e protezione di vittime e testimoni}

Qualche volta, nella corsa verso il raggiungimento d'obiettivi quali la persecuzione dei trafficanti, gli Stati focalizzano la propria attenzione sulle vittime solo per le informazioni che queste sono in grado di fornire e dunque per la loro utilità nel perseguimento della giustizia penale, rischiando di trattarle come delle mere pedine e non come esseri umani che necessitano protezione ed assistenza.

L'articolo 25 della Convenzione sul Crimine Transnazionale Organizzato richiede agli Stati di adottare misure adeguate per fornire assistenza e protezione alle vittime dei reati previsti dalla stessa, nei casi particolari di minacce intimidatorie o di vendetta ${ }^{36}$. Sollecita, inoltre, la creazione di procedure per assicurare alle vittime l'accesso alle restituzioni ed al risarcimento per il torto subito ed incita ad instaurare dei metodi che facilitino la partecipazione delle vittime ai procedimenti penali contro i trafficanti. Il Protocollo sulla Tratta richiede agli Stati d'origine e di destinazione di adottare misure per

\footnotetext{
${ }^{35}$ Articolo 26: «(I) Ciascuno Stato Parte adotta adeguate misure per incoraggiare persone che partecipino o che abbiano partecipato a gruppi criminali organizzati: a) a fornire alle autorità competenti informazioni utili per scopi investigativi e probatori su questioni quali: i) l'identità, natura, composizione, struttura, collocazione e attività di gruppi criminali organizzati; ii) i collegamenti, compresi quelli internazionali, con altri gruppi criminali organizzati; iii) i reati che i gruppi criminali organizzati abbiano commesso o intendano commettere; b) a fornire alle autorità competenti un aiuto concreto ed effettivo che possa contribuire a privare i gruppi criminali organizzati delle loro risorse o dei proventi di reato. (2) Ciascuno Stato Parte valuta la possibilità, in casi specifici, di attenuare la pena prevista per un imputato che fornisca una cooperazione sostanziale nelle indagini o nel perseguimento di un reato trattato dalla presente Convenzione. (3) Ciascuno Stato Parte valuta la possibilità, nel rispetto dei principi fondamentali delle proprie leggi nazionali, di garantire l'immunità dall'azione penale a chiunque fornisca una collaborazione sostanziale nelle indagini o determini il perseguimento di un reato trattato dalla presente Convenzione. (4) La protezione di tali persone si attua secondo quanto previsto dall'articolo 24 della presente Convenzione. (5) Laddove una persona di cui al paragrafo i del presente articolo che si trova in uno Stato Parte possa fornire una collaborazione sostanziale alle autorità competenti di un altro Stato Parte, gli Stati Parte interessati possono prendere in considerazione la possibilità di stipulare accordi o intese, nel rispetto delle proprie leggi nazionali, in relazione alla possibilità, per l'altro Stato Parte, di disporre il trattamento descritto nei paragrafi 2 e 3 del presente articolo».

${ }^{36}$ Articolo 25: «(I) Ciascuno Stato Parte adotta le misure appropriate nell'ambito dei propri mezzi per fornire assistenza e protezione alle vittime dei reati di cui alla presente Convenzione, in particolare nei casi di minaccia, ritorsione o intimidazione. (2) Ciascuno Stato Parte stabilisce procedure adeguate per consentire il diritto all'indennizzo ed al risarcimento alle vittime dei reati trattati nella presente Convenzione. (3) Ciascuno Stato Parte, nel rispetto delle proprie leggi nazionali, consente che siano esposti gli interessi e le opinioni delle vittime e siano considerati in una fase adeguata dei procedimenti penali contro gli imputati in modo tale da non pregiudicare $i$ diritti della difesa».
} 
favorire il recupero fisico, psicologico e sociale delle vittime di tratta. L'UNODC ha creato un Handbook on Justice for Victims of Crime and Abuse of Power, come strumento utile a dare attuazione ai programmi di supporto per le vittime ${ }^{37}$.

\section{Identificazione delle vittime}

La precoce identificazione delle persone trafficate è un prerequisito indispensabile per riconoscerle come vittime e, conseguentemente, assicurare loro assistenza e protezione. L'United Nations Declaration of Basic Principles of Justice for Victims of Crime and Abuse of Power (1985) stabilisce che i soggetti passibili di entrare in contatto con le vittime (come ad esempio, gli agenti di polizia, il personale sanitario e dei servizi sociali, le guardie di frontiera) dovrebbero essere istruiti per imparare a riconoscere le vittime ed essere sensibili alle loro necessità. L'United Nations High Commissioner for Human Rights ha riportato tra i Recommended Principles and Guidelines on Human Rights and Trafficking le indicazioni piú significative per l'identificazione delle vittime di tratta, che dovrebbero essere tenute presenti per lo sviluppo delle strategie nazionali $i^{38}$.

Sono stati, inoltre, elaborati molti strumenti pratici di gran supporto nel processo d'identificazione delle vittime, tra cui guide per incaricati di servizi sanitari, checklists, tools per agenti di polizia e di altre agenzie governative ${ }^{39}$, raccomandazioni per una condotta etica e sicura nell'interrogatorio delle vittime ${ }^{40}$ e sono state, infine, create delle linee dirette cui le vittime possono chiamare per ricevere assistenza, spesso anche nella propria lingua.

Al fine di facilitare l'accesso delle vittime ai servizi per esse predisposti, gli Stati, come passo ulteriore, possono creare un sistema che consenta la certificazione dello status di vittima ed il conseguente accesso ad una serie di benefici quali il permesso di residenza, $i$ servizi sanitari, il diritto ad un alloggio temporaneo. Un esempio significativo in tal senso è rappresentato dal victim certification process, gestito dall' U.S. Department of Health and Human Services ${ }^{41}$.

Le persone trafficate sono spesso trattate come criminali più che come vittime; nello Stato di destinazione sono perseguite a causa del loro status irregolare d'immigrazione o di lavoro e, se ricondotte nello Stato d'origine, possono essere perseguite per aver utilizzato documenti falsi, per aver lasciato il Paese illegalmente o per aver lavorato nell'industria del sesso. La criminalizzazione limita, così, l'accesso delle vittime di tratta alla giustizia ed alla protezione e diminuisce la possibilità che queste denuncino i fatti alle

${ }^{37}$ UNODC/CICP, Handbook on Justice for victims, 1999,

<www.unodc.org/pdf/crime/publications/standards_9857854.pdf> [consultato: 03-12-2007].

${ }^{38}$ Cfr.: UNHCR, Recommended Principles and Guidelines on Human Rights and Trafficking, 2002,

$<$ http://www.unhchr.ch/huridocda/huridoca.nsf/eo6a5300f9ofao238025668700518ca4/caf3deb 2bo5d4f35ci256bf30051a003/\$FILE/No240168.pdf> [consultato: 03-I2-2007].

${ }^{39}$ A titolo di esempio: U.S.DEPARTMENT OF HEALTH AND HUMAN SERVICES, Screening Tool for victims of human trafficking,

<www.acf.hhs.gov/trafficking/campaign_kits/tool_kit_law/screen_questions.html> [consultato:03-12-2007].

${ }^{40}$ WORLD Health ORganization, Ethical and safety recommendations for interviewing trafficked women, 2003,

<http://www.who.int/gender/documents/en/final\%2orecommendations\%2023\%2ooct.pdf>, [consultato:03-I2-2007].

${ }^{4 r}$ U.S Department of Health AND HUMAn SeRvices, Certification for victims of trafficking, <www.acf.hhs.gov/trafficking/about/cert_victims.html> [consultato:03-I2-2007]. 
autorità competenti: alla paura per la propria sicurezza personale e per la rappresaglia da parte dei trafficanti, si aggiunge, infatti, in queste condizioni, la paura di essere perseguite e condannate.

Tuttavia, né la Convenzione sul Crimine Transnazionale Organizzato né il Protocollo sulla Tratta contengono una specifica previsione che obblighi gli Stati a non criminalizzare le vittime di tratta. Esistono, invece, una serie di dichiarazioni e risoluzioni non vincolanti che affrontano il tema in modo specifico tra cui l'Hague Ministerial Declaration che avverte gli Stati sulla necessità di assicurarsi che nessuna azione, inclusa la detenzione, che possa mettere a repentaglio la testimonianza di una vittima in un procedimento penale, sia intrapresa per ragioni d'immigrazione. Inoltre, alcuni Stati hanno provveduto alla creazione di un sistema d'armonizzazione delle leggi nazionali in modo tale che includano il principio di non-criminalizzazione delle persone trafficate ed un espresso divieto di internare le stesse in centri di detenzione. Per esempio, in Kosovo la legge permette alle persone trafficate di usare la tratta come elemento di difesa nei casi di persecuzione per prostituzione o entrata illegale nel Paese. Nonostante tale prospettiva tenga in considerazione i diritti umani, le persone trafficate continuano ad essere accusate di reati commessi durante il periodo in cui sono vittime. Ove ciò accada ed in assenza di leggi che lo impediscano, le vittime dovrebbero almeno poter sollevare, in propria difesa, l'utilizzo da parte dei trafficanti di mezzi di coercizione fisica o psicologica o di minaccia dell'uso della forza nel momento in cui il reato è stato commesso, essendo tali principi universalmente riconosciuti.

\section{Ambiti di protezione}

Le attività di protezione di vittime e testimoni del reato di tratta sono molteplici, si esplicano in diversi ambiti e sono poste in essere da differenti soggetti. Chiunque entri in contatto con vittime di tratta, nello svolgimento delle proprie funzioni, ha l'imprescindibile dovere giuridico ed umanitario di agire nel rispetto dei loro fondamentali diritti umani, in qualunque stato della fase investigativa e processuale ed anche successivamente. Gli investigatori devono, in particolare, informare le vittime di tutte le conseguenze ed i rischi ai quali si espongono nel momento in cui decidono di prestare un certo tipo di collaborazione. L'accesso all'informazione è, infatti, il punto di partenza per la partecipazione delle vittime ai procedimenti penali; per tale ragione, il Protocollo sulla Tratta richiede agli Stati di fornire alle vittime informazioni sul loro ruolo nei procedimenti penali ed amministrativi nonché sui loro diritti, in una lingua per esse comprensibile. Inoltre, non disponendo molte vittime dei mezzi finanziari per pagare un avvocato, è importante che gli Stati forniscano servizi d'assistenza e rappresentanza legale gratuita, anche mediante accordi con organizzazioni non governative specializzate. È ancora compito degli investigatori informare le vittime di tutti i servizi di supporto esistenti, offerti da organizzazioni governative e non e mettere in contatto le vittime con tali organizzazioni.

I servizi disponibili sono, in accordo con quanto stabilito dal Protocollo sulla Tratta, di vario tipo. In primo luogo, le vittime hanno spesso bisogno d'immediata consulenza medica, una volta giunte nel Paese di destinazione o ritornate in quello d'origine, perché hanno riportato delle lesioni, perché sono state esposte al rischio di malattie o perché possono essere state costrette all'assunzione di droghe da parte di chi ha avuto necessità di tenerle sotto controllo. I programmi di assistenza medica alle vittime di tratta possono beneficiare della collaborazione degli uffici regionali della World Health Organization (WHO). In secondo luogo, le vittime necessitano di programmi d'assistenza psicologica che le aiutino a riprendersi dal terribile trauma dell'esperienza di tratta (che genera ansietà, depressione, paura, sensi di colpa, perdita di fiducia negli altri, etc.) ed a ricominciare una vita normale, soprattutto sotto il profilo delle relazioni interpersonali. Inoltre, nell'offerta di servizi di supporto alle vittime bisogna tener conto dell'esigenza di 
fornire loro assistenza linguistica, principalmente mediante traduzioni del materiale informativo ed interpreti, in modo che queste possano sempre rendersi conto dei procedimenti in cui sono coinvolte. Infine le vittime hanno bisogno di un rifugio sicuro, per breve o per lungo tempo, dove poter vivere al riparo dalle minacce dei trafficanti.

Sotto altro profilo, considerando che le agenzie di controllo delle frontiere sono prive di personale, infrastrutture e fondi adeguati per procedere ad ispezioni efficaci, che le nuove tecnologie consentono la falsificazione di documenti su larga scala e che la corruzione è una piaga ancora tanto diffusa, è d'essenziale importanza che gli Stati provvedano all'adozione di misure per ostacolare lo spostamento di persone attraverso i confini da parte dei trafficanti. In proposito, l'articolo II del Protocollo sulla Tratta richiede agli Stati membri di rafforzare i controlli alle frontiere e la cooperazione tra gli agenti dei diversi Paesi, mediante la creazione di canali diretti di comunicazione ${ }^{42}$. L'articolo I2 richiede agli Stati di assicurarsi che i documenti d'identità e di viaggio emessi non siano facilmente alterabili o falsificabili $i^{43}$. L'articolo I3 prevede che, su richiesta di altro Stato, uno Stato debba, in tempo ragionevole, verificare la legittimità e la validità di documenti che si suppone siano stati emessi dallo Stato stesso ed utilizzati a scopo di tratta ${ }^{44}$.

La Convenzione sul Crimine Transnazionale Organizzato dedica, inoltre, alcuni articoli all'argomento specifico della protezione dei testimoni. L'articolo 24 richiede agli Stati membri l'adozione di misure adeguate per fornire effettiva protezione da forme d'intimidazione o vendetta a coloro che intendono prestare testimonianza (nonché ai loro parenti e prossimi congiunti), all'interno di procedimenti penali, in relazione ai reati previsti dalla Convenzione ${ }^{45}$. Tali misure possono includere la protezione fisica di questi

\footnotetext{
${ }^{42}$ Articolo II: «(I) Senza pregiudizio per gli impegni internazionali relativi alla libera circolazione delle persone, gli Stati Parte potenziano, nella misura del possibile, i controlli alle frontiere necessari per prevenire e individuare la tratta di persone. (2) Ogni Stato Parte adotta misure legislative o altre misure adeguate per impedire, nella misura del possibile, che i mezzi di trasporto utilizzati dai trasportatori commerciali siano utilizzati nella commissione dei reati di cui all'articolo 5 del presente Protocollo. (3) Laddove opportuno, e senza pregiudizio per le convenzioni internazionali applicabili, tali misure includono la determinazione dell'obbligo per i trasportatori commerciali, inclusi qualsiasi compagnia di trasporto o il proprietario o gestore di qualsiasi mezzo di trasporto, di verificare che tutti passeggeri siano in possesso dei documenti di viaggio richiesti per l'ingresso nello Stato di accoglienza. (4) Ogni Stato Parte adotta le misure necessarie, in conformità con il proprio diritto interno, per prevedere sanzioni nei casi di violazione dell'obbligo di cui al paragrafo 3 del presente articolo. (5) Ogni Stato Parte prende in considerazione l'adozione di misure che consentano, in conformità con il proprio diritto interno, di rifiutare l'ingresso delle persone coinvolte nella commissione dei reati di cui al presente Protocollo o di ritirare i loro visti. (6) Senza pregiudizio per l'articolo 27 della Convenzione, gli Stati Parte prendono in considerazione il rafforzamento della cooperazione tra gli organismi di controllo delle frontiere, anche tramite la costituzione e il mantenimento di canali diretti di comunicazione».

${ }^{43}$ Articolo I2: «Ogni Stato Parte adotta le misure necessarie, secondo i mezzi disponibili, al fine di: a) assicurare che $i$ documenti di viaggio o di identità da esso rilasciati siano di una qualità tale da non poter essere facilmente utilizzati in maniera impropria e da non poter essere facilmente falsificati o illegalmente modificati, duplicati o rilasciati; b) assicurare l'integrità e la sicurezza dei documenti di viaggio e di identità rilasciati da o per conto dello Stato Parte e impedire che siano creati, rilasciati ed utilizzati illegalmente».

${ }^{44}$ Articolo I3: «Su richiesta di un altro Stato Parte, uno Stato Parte, in conformità con il proprio diritto interno, verifica entro un lasso di tempo ragionevole, la legittimità e la validità dei documenti di viaggio e di identità rilasciati, o che si presume siano stati rilasciati in suo nome e sospettati di essere utilizzati per la tratta di persone».

${ }^{45}$ Articolo 24: «(I) Ciascuno Stato Parte adotta misure adeguate, nell'ambito dei propri mezzi, per garantire efficaci forme di protezione da potenziali ritorsioni o intimidazioni dei testimoni che depongono in processi penali in relazione a reati di cui alla presente Convenzione e, qualora necessario, dei loro familiari e delle altre persone ad essi vicine. (2) Le misure previste dal paragrafo I del presente articolo possono includere, tra le altre, fatti salvi i diritti dell'imputato, compreso il
} 
soggetti, il trasferimento degli stessi, limitazioni al rilascio d'informazioni riguardanti la loro identità o altre notizie rilevanti oppure consistere nell'emanazione di regole processuali che permettono loro di rendere testimonianza con modalità che ne salvaguardano la sicurezza (ad esempio prevedendo che non ne sia rivelata l'identità o predisponendo la resa della testimonianza in video-conferenza ${ }^{46}$ ). Le previsioni enunciate sono obbligatorie, ma solo «where appropriate» (cioè limitatamente a casi e condizioni specifiche) e «within the means» (cioè tenendo conto delle risorse disponibili e delle capacità tecniche) dello Stato in questione. La Convenzione affronta, infine, nell'articolo 23, la questione dell'ostruzione della giustizia, criminalizzando sia i casi di ricorso a mezzi di corruzione (come ad esempio la bustarella) sia i casi di ricorso a mezzi coercitivi quali l'uso o la minaccia di violenza ed indipendentemente da che ciò avvenga nella fase pre-processuale o in quella propriamente processuale $^{47}$.

3. Status di residenza, rimpatrio e reintegrazione delle vittime

Le persone trafficate non godono, il più delle volte, di uno status di residenza regolare nello Stato di destinazione, o perché sono arrivate illegalmente o perché il loro permesso di residenza è scaduto. L'articolo 7 del Protocollo sulla Tratta incoraggia gli Stati all'adozione di strumenti legislativi che consentano alle vittime di ottenere un permesso di residenza temporaneo o permanente nel territorio dello Stato ${ }^{48}$. Esempi di good practices, in tale direzione, sono offerti dalle legislazioni belga, olandese, inglese e statunitense. È indicativo, inoltre, che il Consiglio dell'Unione Europea abbia emanato una Direttiva sul rilascio del titolo di soggiorno ai cittadini di Paesi terzi vittime di tratta che collaborano con le autorità competenti ${ }^{49}$. Questo tipo di misure hanno sortito, da un lato, un effetto notevole

diritto al giusto processo: a) l'istituzione di procedure per la protezione fisica di tali persone quali, nella misura necessaria ed attuabile, il trasferimento il domicilio e permettendo, laddove possibile, il divieto o la limitazione di accesso alle informazioni concernenti l'identità e la dislocazione di tali persone; b) l'adozione di norme relative alle prove onde permettere che le deposizioni siano rese in maniera tale da assicurare l'incolumità del testimone anche attraverso l'uso di mezzi tecnologici di comunicazione come collegamenti video ed altri strumenti adeguati. (3) Gli Stati Parte valutano la possibilità di stipulare accordi o intese con altri Stati per il trasferimento del domicilio delle persone di cui al paragrafo I del presente articolo. (4) Le disposizioni del presente articolo si applicano anche alle vittime in quanto testimoni».

${ }^{46}$ A titolo di esempio, in Italia la via che è stata adottata per proteggere la sicurezza dei testimoni è di far rendere loro testimonianza mediante «incidente probatorio», vale a dire mediante un mezzo incidentalmente inserito nella fase investigativa o dell'udienza preliminare, che prevede un'udienza «in camera di consiglio».

${ }^{47}$ Articolo 23: «Ciascuno Stato Parte adotta misure legislative o di altra natura che possono essere necessarie a conferire il carattere di reato, quando commesso intenzionalmente: a) all'uso della forza fisica, minacce o intimidazioni o alla promessa, offerta o concessione di vantaggi considerevoli per indurre falsa testimonianza o per interferire in deposizioni testimoniali o nella produzione di prove nel corso di processi relativi alla commissione di reati di cui alla presente Convenzione; b) all'uso della forza fisica, minacce o intimidazioni per interferire con l'esercizio di doveri d'ufficio da parte di un magistrato o di un appartenente alle forze di polizia in relazione alla commissione di reati di cui dalla presente Convenzione. Nulla nella presente lettera pregiudica il diritto degli Stati Parte di avere una legislazione che protegga altre categorie di pubblici ufficiali».

${ }^{48}$ Articolo 7: «(I) Oltre alle misure di cui all'articolo 6 del presente Protocollo, ogni Stato Parte prende in considerazione l'adozione di misure legislative o di altre misure adeguate che consentano alle vittime della tratta di persone di restare sul suo territorio, a titolo temporaneo o permanente, nei casi opportuni. (2) Nell'attuare le disposizioni di cui al paragrafo I del presente articolo, ogni Stato Parte tiene debitamente conto dei fattori umanitari e personali».

${ }^{49}$ CONSIGlio Dell'Unione EUROPEA, Direttiva 2004/81/CE del 29 aprile 2004 riguardante il titolo di soggiorno da rilasciare ai cittadini di Paesi terzi vittime della tratta o coinvolti in un'azione di 
sulle vittime, le quali si sono mostrate più disponibili a testimoniare contro i trafficanti e dall'altro, hanno facilitato il lavoro delle organizzazioni non governative che incoraggiano le vittime cui offrono servizi a denunciare gli avvenimenti alle autorità governative.

L'articolo 8 del Protocollo stabilisce che gli Stati devono facilitare il rimpatrio delle vittime di tratta che sono cittadini o hanno il permesso di residenza nello Stato, ed a tal fine, devono provvedere a fornire, su richiesta di altro Stato ed in tempi ragionevoli, i documenti di viaggio necessari ${ }^{50}$. Alcuni Stati hanno firmato, in aggiunta, accordi bilaterali che disciplinano il processo amministrativo di rimpatrio e le organizzazioni non governative stanno assumendo un ruolo sempre più incisivo nell'assistenza alle vittime durante la fase di rimpatrio. Un decisivo passo in avanti sarebbe rappresentato dallo sviluppo di accordi tra i Paesi di destinazione e quelli d'origine in modo tale da assicurare alle vittime che sarà loro fornita assistenza anche una volta tornate a casa. Le vittime necessitano aiuto non solo per il rimpatrio, ma anche per la reintegrazione nelle proprie famiglie e comunità, in quanto essa comporta problemi di natura psicologica, familiare, di salute, giuridica e finanziaria ${ }^{5 \mathrm{I}}$. Particolarmente delicato è il caso di ritorno di bambini allo Stato d'origine: il Protocollo sulla Tratta impone, infatti, il ricorso a speciali precauzioni per assicurarsi che il rimpatrio avvenga nell'interesse del bambino e che vi sia, nello Stato d'origine, una famiglia o altro ente che si faccia carico della responsabilità di fornirgli cura e protezione. L'UNHCR ha elaborato, sull'argomento, delle Guidelines on Policies and Procedures in dealing with Unaccompanied Children Seeking Asylum, nelle quali tre scopi emergono sugli altri: promuovere la coscienza sui bisogni speciali dei bambini non accompagnati, in accordo con quanto stabilito dalla Convenzione sui Diritti del Fanciullo, sottolineare l'importanza di un approccio completo ed esaustivo al problema, stimolare lo sviluppo di politiche e pratiche

favoreggiamento dell'immigrazione illegale che cooperino con le autoritá competenti, Gazzetta Ufficiale delle Comunitá Europee,

$<$ http://eur-lex.europa.eu/LexUriServ/LexUriServ.do?uri=CELEX:32004Loo8r:IT:HTML> [consultato:03-12-2007].

${ }^{50}$ Articolo 8: «(I) Lo Stato Parte di cui la vittima della tratta di persone è cittadina, o in cui la persona aveva il diritto di risiedere a titolo permanente al momento del suo ingresso nello Stato Parte d'accoglienza, facilita e accetta, tenendo debitamente conto dell'incolumità di questa persona, il ritorno di quest'ultima senza ingiustificato motivo o irragionevole ritardo. (2) Quando uno Stato Parte fa ritornare una vittima della tratta di persone in uno Stato Parte di cui questa persona è cittadina o in cui questa aveva, all'epoca del suo ingresso nel territorio dello Stato Parte d'accoglienza, il diritto di risiedere a titolo permanente, questo ritorno è assicurato tenendo debitamente conto dell'incolumità della persona, nonché dello stato del procedimento penale connesso al fatto che quella persona è vittima della tratta di persone, ed è preferibilmente volontario. (3) Su richiesta dello Stato Parte d'accoglienza, lo Stato Parte richiesto, senza ritardi ingiustificati o irragionevoli, verifica se una persona vittima della tratta di persone è suo cittadino o aveva il diritto di risiedere a titolo permanente sul suo territorio all'epoca dell'ingresso nel territorio dello Stato Parte d'accoglienza. (4) Al fine di facilitare il ritorno della vittima della tratta di persone che non possieda l'adeguata documentazione, lo Stato Parte di cui quella persona è cittadina o nel quale aveva il diritto di risiedere a titolo permanente all'epoca del suo ingresso nel territorio dello Stato Parte d'accoglienza, accetta di rilasciare, su richiesta dello Stato Parte d'accoglienza, i documenti di viaggio o qualsiasi altra autorizzazione necessaria per permettere alla persona di viaggiare e rientrare nel suo territorio. (5) Il presente articolo non reca pregiudizio a nessuno dei diritti riconosciuti alle vittime della tratta di persone dal diritto interno dello Stato Parte d'accoglienza. (6) Il presente articolo non reca pregiudizio a nessun accordo o intesa bilaterale o multilaterale applicabile che disciplina, in tutto o in parte, il ritorno delle vittime della tratta di persone».

${ }^{51}$ Un'elencazione approfondita delle varie difficoltà che possono incontrare le vittime dopo il rimpatrio è contenuta in SAVE THE CHILDREN, UN-IAP, IOM, Training Manual for Combating Trafficking in Women and Children, elaborato nell'ambito dell'U.N. Inter-Agency Project on Trafficking in Women and Children in the Sub-Mekong Region,

<http://www.un.or.th/TraffickingProject/trafficking_manual.pdf> [consultato:03-I2-2007]. 
che soddisfino i bisogni dei bambini non accompagnati ${ }^{52}$. L'IOM offre, d'altro canto, un programma di supporto per il ritorno volontario di migranti irregolari, i quali vengono assistiti nel recupero dei documenti di viaggio e dei biglietti, nel trasporto e nella ricezione nello Stato d'origine. Non appena rientrati, questi possono iscriversi ad un programma di reintegrazione che offre servizi d'alloggio, consulenza legale e supporto familiare ${ }^{53}$.

È compito degli Stati d'origine, infine, provvedere all'istituzione di programmi di riabilitazione delle vittime, mediante l'inserimento in programmi d'educazione o l'offerta d'opportunità lavorative. Ciò vale anche nel caso in cui le vittime non vengono rimpatriate $\mathrm{e}$ continuano a vivere nello Stato di destinazione ${ }^{54}$.

\section{Restituzioni e risarcimento per le vittime}

Le vittime di tratta hanno diritto ad essere risarcite dal trafficante per il danno fisico o morale subito o perché non hanno ricevuto compenso per il lavoro o i servizi prestati. Sia la Convenzione sul Crimine Transnazionale Organizzato sia il Protocollo sulla Tratta richiedono agli Stati di prevedere delle procedure che permettano alle vittime di ottenere le restituzioni ed il risarcimento: tali procedure possono consistere nella possibilità di promuovere azione giudiziaria contro i trafficanti ed i loro complici per ottenere la riparazione dei danni civili, nella possibilità che i tribunali ordinino il risarcimento o le restituzioni a quanti sono condannati per i reati in questione o ancora nell'istituzione di fondi dello Stato cui le vittime possono attingere se hanno subito danni o lesioni in conseguenza di un reato. Al fine di garantire l'accesso al risarcimento non è sufficiente l'esistenza di una previsione legislativa, occorrendo anche la previa informazione alle vittime circa il loro diritto al risarcimento per opera della polizia o di avvocati privati nonché la confisca dei proventi del reato e l'utilizzo degli stessi a fini risarcitori. Alcuni Stati forniscono un'ulteriore possibilità di risarcimento mediante la creazione di fondi sussidiari che consentono alle vittime di richiedere denaro allo Stato quando il risarcimento non può essere ottenuto dal trafficante. In genere, hanno accesso a tale tipo di fondi non delle categorie particolari di vittime, ma tutte quelle che, in conseguenza di un reato, hanno subito un danno fisico, sessuale o all'integrità morale. Questi fondi possono essere variamente finanziati attraverso multe (come in Austria, in Belgio ed in molti Stati americani), confisca di proprietà dei colpevoli (Kosovo), tasse (Canada, Francia, Inghilterra), altri mezzi statali (Finlandia, Olanda, Emirati Arabi Uniti) o donazioni d'istituzioni ed individui privati (Polonia). Requisito indispensabile per usufruire di questi fondi è, tuttavia, lo status di cittadino o residente permanente, cosa che ne esclude l'accesso a quelle vittime che rimangono negli Stati di destinazione.

${ }^{52}$ UNHCR, Guidelines on Policies and Procedures in dealing with Unaccompanied Children Seeking Asylum, I997, <http://www.unhcr.org/publ/PUBL/3d4f9Icf4.pdf> [consultato:03-I2-2007].

${ }^{53}$ Per maggiore approfondimento si veda InTERnATIONAL MigRATIOnS OfFICE, Return Migration: Policies and Practices in Europe,, 2004,

<http://www.iom.int/jahia/webdav/site/myjahiasite/shared/shared/mainsite/published_docs/bo oks/return_migration.pdf> [consultato:03-12-2007].

${ }^{54}$ In Italia, per esempio, esiste un programma sponsorizzato dal Ministero delle Pari Opportunità e gestito dal Servizio Migranti Caritas di Torino, che si occupa della riabilitazione delle vittime di tratta e mira a rendere le stesse indipendenti mediante il lavoro. Durante il programma, le vittime frequentano corsi di lingua italiana e ricevono il supporto di un assistente sociale e di uno psicologo. 


\section{Prevenzione del fenomeno di tratta}

Il Protocollo sulla Tratta esorta gli Stati ad intraprendere politiche e programmi atti a prevenire e combattere il fenomeno ed a proteggere le vittime, specialmente le donne ed i bambini, dal rischio di cadere nelle mani dei trafficanti. In particolare, si suggeriscono misure quali ricerche, campagne d'informazione attraverso i mass media e soprattutto iniziative economiche e sociali volte ad incidere sui fattori (povertà, mancanza di opportunità, conflitti armati, discriminazione nei confronti delle donne) che contribuiscono a generare il desiderio di emigrazione e la conseguente vulnerabilità delle vittime. Più specificamente, le campagne di sensibilizzazione dell'opinione pubblica sui mali associati al fenomeno di tratta, portate avanti essenzialmente attraverso i mezzi d'informazione, possono rivelarsi degli strumenti piuttosto efficaci. L'UNODC, ad esempio, ha dato vita ad alcuni annunci pubblicitari che hanno registrato un gran successo ${ }^{55}$. Le campagne devono essere formulate in maniera accessibile per le vittime, usando materiale e linguaggio adeguato al target dei destinatari; esse forniscono informazioni sui rischi della tratta, sulle false promesse di emigrazione legale, di lavoro e di facili guadagni ed ancora sui rischi di contrazione di malattie o di disagi fisici connessi con l'attività di sfruttamento sessuale. Molte campagne d'informazione sono, inoltre, portate avanti da organizzazioni non governative ed hanno come destinatari gli adolescenti ed i giovani ${ }^{56}$.

Si richiede inoltre, ai fini di un intervento più efficace, da un lato, la collaborazione con organizzazioni non governative e con altri elementi della società civile e dall'altro, la conclusione di accordi bilaterali o multilaterali per strategie d'azione comuni volte, per esempio, a scoraggiare la domanda di tutte le forme di sfruttamento di persone ${ }^{57}$.

Non è da sottovalutare il ruolo dei funzionari corrotti nello schema del reato di tratta. La Convenzione sul Crimine Transnazionale Organizzato crea, per gli Stati, obblighi specifici di criminalizzazione della corruzione nelle sue diverse forme di «active bribery» (pagamento di una bustarella), «passive bribery» (accettazione di una bustarella) e partecipazione in qualità di complice ad un'azione di corruzione. L'UNODC ha anche preparato un anti-corruption toolkit che contiene delle linee guida importanti per chiunque si trovi faccia a faccia con un caso di corruzione ${ }^{58}$.

Riguardo alle politiche volte a scoraggiare la domanda di sfruttamento di persone trafficate, allo stato attuale, non è sempre facile raggiungere una posizione d'accordo tra gli

\footnotetext{
${ }_{55}$ Alcuni annunci pubblicitari riguardano la tratta a scopo di sfruttamento sessuale e di lavoro forzato ed altri ritraggono donne di differenti nazionalità che compiono il passo di cercare supporto. Si tratta di video della durata di trenta, quarantacinque o sessanta secondi che sono diffusi attraverso le reti regionali e nazionali e contenenti, nella schermata finale, l'indicazione di un numero diretto per l'assistenza.

Per visionare gli spots: <www.unodc.org/unodc/multimedia.html> [consultato:03-I2-2007].

${ }^{56}$ A titolo di esempio, si veda il lavoro condotto dall'ONG La Strada, mediante lezioni e seminari nei Paesi dell'Europa centro-orientale, <http://www.lastradainternational.org/?main=home> [consultato:03-I2-2007].

${ }^{57}$ Un esempio rilevante d'accordo bilaterale, in armonia con l'enunciazione del Protocollo sulla Tratta, è rappresentato da quello firmato tra l'Italia e l'ufficio dell'IOM di Tirana in Albania allo scopo di gestire i flussi di migranti dall'Albania e di favorire l'integrazione sociale e professionale degli stessi in Italia. Esso ha consentito a cinquemila albanesi di ottenere un contratto di lavoro in Italia per un anno, non appena giunti a destinazione. L'ufficio dell'IOM di Tirana si è occupato di selezionare gli aspiranti, sulla base di test linguistici e professionali e di inserirli in una banca dati, accessibile via Internet alle imprese italiane in cerca di lavoratori.

${ }^{58}$ UNODC, U.N. Anti-Corruption Toolkit, 2004

$<$ http://www.unodc.org/pdf/corruption/publications_toolkit_sepo4.pdf> [consultato:03-I22007].
} 
Stati. Se prendiamo l'esempio della prostituzione, infatti, alcuni Stati, come ad esempio l'Olanda, ritengono che la legalizzazione del mercato del sesso possa rappresentare una soluzione appropriata a ridurre la domanda ed a migliorare le condizioni di lavoro delle donne, mentre altri ritengono, al contrario, che l'esistenza di case d'appuntamento legalizzate possa creare una domanda costante di nuovo reclutamento ed alimentare il mercato illegale della tratta. Una politica che va certamente favorita è quella della creazione di leggi, istituzioni e programmi destinati ad eliminare le discriminazioni basate sul sesso ed a promuovere i diritti economici delle donne. La Bulgaria ha, ad esempio, realizzato un progetto di tre anni, finanziato dall'UNDP, per promuovere l'arricchimento economico delle donne e produrre posti di lavoro con accesso riservato, in via preferenziale, alle donne.

L'iniziativa piú significativa promossa dall'UNODC ai fini della prevenzione del fenomeno di tratta è certamente l'United Nations Global Initiative to Fight Human Trafficking (UN.GIFT), lanciata nel marzo del 2007 in collaborazione con l'ILO, l'IOM, l'UNICEF, l'UNHCR e l'OSCE. Tale iniziativa si prefigge essenzialmente i seguenti scopi: incrementare la coscienza sul fenomeno di tratta, ampliare la conoscenza di dati, fatti e statistiche sulla tratta attraverso una migliore raccolta, analisi e condivisione dei dati disponibili ed il lavoro di squadre di ricerca congiunte, migliorare l'assistenza tecnica ai Governi ed agli altri attori coinvolti a livello locale, regionale ed internazionale ${ }^{59}$.

\section{Progetti di assistenza tecnica}

I Progetti di assistenza tecnica costituiscono uno dei punti chiave del GPAT e sicuramente la parte più consistente delle attività in cui si concreta il Programma: mediante tali progetti di cooperazione si cerca di dare concreta attuazione alle previsioni della Convenzione sul Crimine Transnazionale Organizzato e del Protocollo sulla Tratta che costituiscono la base normativa dell'intero Programma Globale delle Nazioni Unite. A livello regionale e nazionale, in dipendenza dai risultati delle valutazioni sulle dimensioni del fenomeno e sulla capacità delle forze locali di contrastarlo, si seleziona uno dei moduli d'intervento elaborati in accordo con il contenuto basico del Protocollo. I moduli dei progetti d'assistenza tecnica sono i seguenti: pre-ratification assistance; criminal justice system responses; victim protection and support; international cooperation; prevention and awarenessraising.

Il primo modulo di pre-ratification assistance comporta l'apprezzamento della legislazione esistente sulla tratta, l'elaborazione di un report di valutazione sui requisiti necessari per la ratifica del Protocollo e l'instaurazione di un dialogo tra le autorità locali e le organizzazioni non governative allo scopo di dare attuazione al Protocollo. Questo tipo d'attività è portato avanti in collaborazione con il Segretariato della Commissione del Centre for International Crime Prevention e con il Legal Affairs Branch dell'UNODC. In collaborazione con tali entità viene realizzato anche il secondo modulo di criminal justice system responses, mediante il quale si provvede all'adozione di misure legislative che criminalizzano la tratta, all'organizzazione di sessioni di training per i professionisti chiamati ad operare sul campo ed allo sviluppo di speciali tecniche d'indagine nonché di canali che consentano un rapido scambio d'informazioni tra le agenzie coinvolte nell'azione. Il terzo modulo di victim protection and support si prefigge la creazione di servizi di supporto per le vittime che includono l'assistenza fisica, psicologica e sociale, il risarcimento per i danni sofferti, i programmi di recupero per le vittime rimpatriate, i servizi d'offerta d'alloggi. Il quarto modulo di international cooperation, in attuazione di quanto previsto dalla Convenzione sul Crimine Transnazionale Organizzato e dal Protocollo sulla Tratta, si propone di rafforzare la collaborazione tra i professionisti della

\footnotetext{
${ }^{59}$ Per maggiore approfondimento si veda <http://www.ungift.org/> [consultato:03-I2-2007].
} 
giustizia penale dei diversi Paesi, nelle fasi d'indagine e di persecuzione dei colpevoli e di rafforzare i controlli delle frontiere per prevenire il fenomeno. Il quinto modulo di prevention and awareness-raising, infine, prevede la messa in atto di programmi e misure varie per prevenire e combattere il fenomeno di tratta e per impedire la re-vittimizzazione delle persone trafficate. Questo tipo d'attività richiede la collaborazione di agenzie governative e non governative. È chiaramente possibile che l'intervento in un determinato Paese si realizzi attraverso la messa in atto di diversi moduli.

Per quanto riguarda il project life cycle, un vero e proprio Project Cycle Management dell'UNODC non è ancora disponibile e dunque la Division for Operations si serve di una serie di strumenti messi insieme nella prassi durante il corso degli anni ${ }^{60}$. Sinteticamente le fasi del project life cycle sono quelle evidenziate nel grafico riportato di seguito e cioè la formulazione del progetto, lo sviluppo (da elaborarsi in collaborazione con i finanziatori), l'approvazione, l'esecuzione e la valutazione finale.

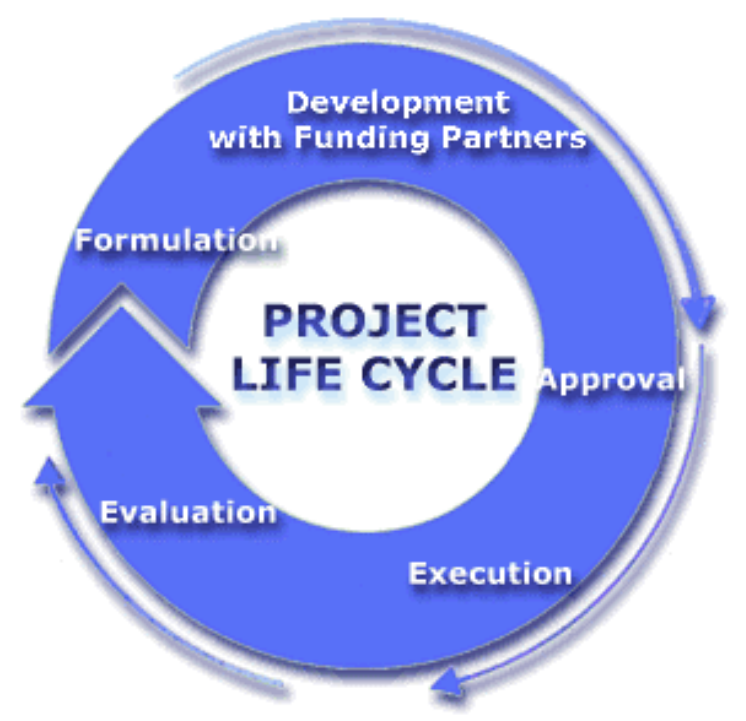

La fase di formulazione (che presuppone un'operazione di programmazione, con l'indicazione delle priorità operative in riferimento al profilo di ciascun Paese) comincia con l'elaborazione della project idea, che proviene normalmente dal Field Office e che contiene una prima stima del budget necessario per portare avanti il progetto e si conclude con l'approvazione del project document (contenente l'indicazione definitiva del budget ed una check list delle modalità d'esecuzione) da parte del Direttore Esecutivo dell'UNODC, su indicazione del Programme and Project Committee (PPC). La seconda fase, portata avanti principalmente dagli Headquarters Offices, serve a procurare i fondi dai potenziali donatori ed a questa segue la fase d'approvazione definitiva da parte del PPC. La fase d'esecuzione, portata avanti dal Field Office, è coordinata dal Regional Desk, anche mediante input provenienti dagli Headquarters Offices. Essa implica la conclusione d'accordi con agenzie esecutive associate, il reclutamento del personale e l'approvvigionamento. Durante la fase finale di valutazione interviene un external evaluator, incaricato appunto di dare un giudizio di valore su ogni aspetto dell'esecuzione e vengono inoltre elaborati una serie di reports finali esplicativi dell'esecuzione e dei risultati dell'azione.

All'interno del GPAT, a partire dal I999, più di trenta progetti d'assistenza tecnica hanno avuto inizio in Paesi dell'Africa, dell'Asia, dell'Europa e del Latino America. Tra

6o L'Independent Evaluation Unit della Division for Policy Analysis and Public Affairs dell'UNODC sta lavorando ad un progetto di revisione dello stesso [dato aggiornato al febbraio 2006]. 
questi, solo pochi sono già stati completati (essendo anche intervenuta la fase di valutazione), la maggioranza è ancora in corso ed i restanti sono inclusi nell'attuale workplan, anche se non sono ancora stati iniziati ${ }^{6}$. Tra quelli in corso ve ne sono alcuni che si differenziano dagli altri perché non sono destinati ad un Paese o ad una Regione specifici, ma affrontano la problematica della tratta a livello mondiale e perciò sono detti «globali». Tra essi, oltre al progetto di creazione di un database generale sui flussi della tratta non ancora terminato, vi è un importante progetto di elaborazione di un Toolkit to Combat Trafficking in Persons terminato nell'ottobre del 2006, che costituisce una guida pratica contenente le best practices per i Governi, le organizzazioni non governative, gli agenti di polizia e gli altri attori direttamente coinvolti nella lotta contro la tratta di esseri umani $^{6_{2}}$.

\section{Monitoraggio e valutazione dei progetti e dei programmi}

Il monitoraggio e la valutazione sono momenti essenziali del ciclo di vita di un progetto o di un programma. Il primo consiste nell'osservazione costante o perlomeno regolare dello stato d'evoluzione degli stessi e serve per rifinire le strategie d'azione in corso mediante l'identificazione e la risoluzione di problemi che potrebbero incidere negativamente sui risultati definitivi. La seconda, invece, consiste in una riflessione sistematica in cui si misurano oggettivamente, in termini di rilevanza, efficienza ed impatto, i risultati raggiunti mediante i progetti o programmi. La valutazione è una fase cui devono prender parte tutti i partners di un progetto o di un programma, perché bisogna considerare sia la resa dell'intero progetto o programma sia il contributo di ogni singolo elemento nella resa complessiva. Il primo elemento da accertare è se il progetto o programma è stato eseguito secondo il disegno originario o se sono subentrati aggiustamenti in fase d'esecuzione; il secondo è la verifica del raggiungimento degli obiettivi prefissati mediante l'utilizzo d'indicatori delle tendenze del fenomeno ed il terzo e più difficile è la rilevanza, secondo il principio di causalità e non interferenza d'altri fattori, dei collegamenti esistenti tra i risultati raggiunti e gli interventi previsti dal disegno iniziale. Alcuni principi base disciplinano tale fase: innanzitutto è necessario creare una separazione tra la fase di gestione del progetto o programma e quella di valutazione e per assicurare ciò

${ }^{6}$ ON-GOING COUNTRY/REGIONAL PROJECTS:

I) Philippines: Support for Victims/Witnesses of Trafficking in Human Beings. 2) Vietnam: Strengthening of the Legal and Law Enforcement Institutions in Preventing and Combating Trafficking in Persons. 3) West Africa: Measures to Combat Trafficking in Human Beings in Benin, Nigeria, Togo. 4) ECOWAS: Assistance for the implementation of the ECOWAS Plan of Action. 5) Southern Africa: Assistance to law enforcement and prosecutions services. 6) South Africa: Strengthening the fight against Child Trafficking. 7) SADC: Assistance in the formulation and implementation of the SADC Declaration and Plan of Action. 8) Brazil: Measures against Trafficking in Human Beings. 9) Czech Republic and Poland: Criminal Justice Response to Trafficking in Human Beings. Io) Slovakia: Criminal Justice Response to Trafficking in Human Beings. II) Moldova: Building Capacity to Combat Trafficking in Persons. I2) Colombia: Combating Trafficking in Human Beings . 13) Asia/Pacific: Computer- based training module. I4) Lebanon: Measures to prevent and combat Trafficking in Human Beings. I5) Albania: Strengthening border control capacities in Albania. I6) Vietnam: Strengthening of the Legal and Law Enforcement Institutions in Preventing and Combating Trafficking in Persons. I7) Measures to Prevent and Combat Trafficking in Human Beings in the Western African Subregion (Benin, Burkina Faso, Ghana, Niger, Nigeria and Togo). I8) Global/NGOs: Providing support to Victims of Trafficking. I9) Global Database [dati aggiornati al febbraio 2006].

${ }^{62}$ UNODC, Toolkit to Combat Trafficking in Persons, 2006 2007].

$<$ http://www.unodc.org/documents/human-trafficking/HT-toolkit-en.pdf> [consultato:03-I2- 
quest'ultima viene affidata all'Independent Evaluation Unit (IEU) dell'UNODC che, in collaborazione con i Field e gli Headquarter Offices responsabili del progetto o programma, provvede alla selezione degli evaluators. Questi ultimi sono identificati secondo criteri di competenza, imparzialità e integrità morale per assicurare la trasparenza e la massima obiettività delle valutazioni. Inoltre, ciascuna parte del progetto è normalmente invitata a nominare un evaluator o a partecipare alla selezione della squadra di evaluators. I donatori possono essere nominati observers, ma il costo della loro partecipazione alle operazioni deve essere coperto dall'organizzazione che rappresentano. Il capo della squadra di evaluators è nominato dall'UNODC o da altra Agenzia nel caso in cui il contributo finanziario di questa ecceda quello dell'UNODC. La valutazione è dunque affidata ad un organo indipendente mediante il mutuo consenso delle parti ed investe il concept ed il design del progetto, la sua realizzazione ed i risultati raggiunti in termini di impatto e sostenibilità. Comprende, inoltre, le lessons learned e le recommendations nonché l'indicazione delle best practices. Le previsioni che riguardano la valutazione sono contenute nel project document ed approvate dalle parti mediante la firma dello stesso ${ }^{63}$. Tale tipo di valutazione comporta delle attività a livello globale, regionale, sub-regionale e nazionale, spesso realizzate mediante l'intervento congiunto di esperti dell'UNODC e dei Paesi donatori e beneficiari. L'intervento dei donatori deve essere garantito a diversi scopi: affinché ci si impegni a mettere in atto le raccomandazioni che emergono dalla valutazione, affinché la valutazione serva da contributo per la pianificazione dei progetti e programmi futuri, affinché la diffusione dei risultati della valutazione possa dar vita a dibattiti tra i diversi agenti coinvolti nel progetto o programma.

Infine, dato che le risorse di cui si può disporre sono limitate, la valutazione consente di fissare un ordine di priorità tra le attività future e di ottimizzarle. A tal proposito enunciamo brevemente i criteri usati con più frequenza per la progettazione e la selezione di nuovi programmi ed interventi per combattere la tratta d'esseri umani (criteri emersi proprio in seguito alla valutazione dei risultati delle analisi fin qui condotte):

- compatibilità (se gli interventi provocano le reazioni volute sui destinatari);

- efficacia dei costi (confrontando le risorse impiegate ed i risultati raggiunti);

- sostenibilità (degli interventi sul luogo dopo una prima esecuzione);

- trasparenza (delle operazioni amministrative e finanziarie);

- coerenza (con il resto dei progetti e con gli obiettivi generali).

\section{La valutazione del Programma Globale}

L'Independent Evaluation Unit ha condotto una valutazione complessiva del GPAT da ottobre 2004 a gennaio 2005 , servendosi talvolta del proprio staff e talaltra di evaluators esterni e facendo riferimento ad otto progetti di cooperazione tecnica i cui Paesi beneficiari sono Benin, Nigeria, Togo, Brasile, Colombia, Repubblica Ceca, Polonia, India, Filippine, Slovacchia e Vietnam. La metodologia di valutazione ha compreso otto elementi:

- revisione ed analisi di project documents, project evaluations e programme outputs;

- interviste con staff delle Nazioni Unite, rappresentanti dei Paesi donatori e beneficiari, rappresentanti delle organizzazioni governative e non governative;

\footnotetext{
${ }^{63}$ I fondi per la valutazione dei progetti o programmi sono ricavati dai budgets degli stessi.
} 
- lavoro sul campo in nove Paesi;

- analisi comparata di progetti contro la tratta di esseri umani realizzati al di fuori del GPAT da altre agenzie o organizzazioni;

- osservazione e stima di luoghi e servizi anti-tratta (rifugi per le vittime, centri operativi di polizia ed altro);

- esame minuto della struttura gestionale per verificare i rapporti di interconnessione tra le unità dell'UNODC che si occupano di tratta;

- sondaggio presso gli Stati membri per accertare la conoscenza e l'apprezzamento del GPAT da parte degli stessi;

- inputs dei finanziatori.

Lo scopo della valutazione è stato quello di stabilire se ed in quale misura, fino ad oggi, gli obiettivi del GPAT siano stati raggiunti ed in particolare se ed in quale misura il Programma abbia soddisfatto le esigenze dei Paesi beneficiari e le aspettative dei Paesi donatori, se le risorse siano state utilizzate efficacemente, quale impatto abbia avuto il programma e se i benefici dello stesso siano sostenibili.

Riportiamo di seguito le principali risultanze della valutazione e le relative actions o decisions recommended. Il Programma Globale delle Nazioni Unite contro la tratta di persone è oggi considerato, secondo una prospettiva di giustizia penale e da parte degli esperti internazionali, il programma leader delle Nazioni Unite per l'incentivazione della ratifica del Protocollo sulla Tratta e per il perseguimento di una risposta concordata, a livello internazionale, contro il fenomeno della tratta, nonostante il fatto che altre Agenzie dell'ONU realizzino un numero maggiore di progetti di assistenza tecnica anti-tratta al di fuori di esso. Originariamente esso era stato concepito come un programma della durata complessiva di 36 mesi comprensivo di una prima fase di raccolta dati e di una seconda fase costituita da cinque progetti dimostrativi che avrebbero dovuto condurre, con il contributo degli Stati membri, alla formulazione di una Global Strategy da attuare in un secondo momento. Oggi le prime due fasi sono state completate, ma la Global Strategy non è mai stata elaborata ed il piano di lancio del GPAT non è stato ufficialmente modificato. Esso si è trasformato, di fatto, in un programma duraturo dell'UNODC, che ha ricavato il suo mandato dal Protocollo sulla Tratta. Fino ad oggi, al posto di un strategic plan, l'Anti-Human Trafficking Unit si è servita di work plans annuali, profondamente influenzati dagli indirizzi dei Paesi donatori.

Alla luce dei risultati della valutazione in oggetto, il Direttore Esecutivo dell'UNODC ha raccomandato l'adozione di un strategic plan che identifichi gli obiettivi, i Paesi e le Regioni ove urge intervenire e le risorse necessarie. Dalle valutazioni è poi emerso che i donatori dei progetti chiedono la piena copertura del mandato contenuto nel Protocollo sulla Tratta, mediante interventi mirati a contrastare i casi di tratta a scopo di lavoro forzato o di rimozione degli organi. In effetti, a parte il progetto in Benin, Nigeria e Togo ed un video contenuto nei public service announcements (che si occupano di tratta a scopo di sfruttamento del lavoro), la maggioranza dei progetti sono mirati alle vittime dello sfruttamento sessuale e nessuna attività è stata riservata ai casi di tratta per rimozione di organi. Inoltre, si raccomanda che il GPAT si occupi anche del fenomeno di smuggling di migranti, che attualmente non rientra nel mandato specifico di nessuna unità dell'UNODC e dell'ONU in generale; a tal fine è stato proposto di rinominarlo come GPATS (Global Programme against Trafficking and Smuggling). Altra essenziale raccomandazione è quella di dare esecuzione alla Office restructure approvata nel 2003, che prevede un trasferimento di responsabilità, per i progetti di cooperazione tecnica anti-tratta, dall'Anti-Human Trafficking Unit (AHTU) ai Regional Desks ed ai Field Offices. Al momento attuale, però, non è ancora chiaro quali attività dovrebbero essere condotte dall'AHTU, quali dai Regional Desks, quali 
dai Field Offices e quali invece dovrebbero essere condotte congiuntamente. Inoltre si registra una riluttanza dell'AHTU di passare il controllo dei progetti alle unità che operano sul campo, che si evince dal fatto che non sono state organizzate attività di training per $\mathrm{i}$ Desk e Field Offices, ancora incapaci di gestire progetti di tratta perché popolati principalmente di esperti in traffico di droghe (oggetto del mandato originario dell'UNODC). È importante, tuttavia, mettere subito in luce che questa diversa distribuzione delle funzioni potrebbe portare ad una eccessiva regionalizzazione $\mathrm{o}$ nazionalizzazione del GPAT con la perdita di un approccio globale e di expertise globale.

Attualmente sia i progetti di cooperazione tecnica sia altri tipi di attività rimangono in attesa di implementazione per lungo tempo, richiedendo fra l'altro l'impiego delle risorse disponibili, soprattutto in termini di personale responsabile ed impedendo, quindi, di avanzare con nuovi progetti. Si raccomanda, dunque, l'adozione di un Project Cycle Management dell'UNODC, che preveda, ad esempio, che dal momento in cui un project document viene depositato presso l'AHTU possa passare un massimo di dodici mesi per reperire i fondi necessari, trascorsi i quali il progetto deve considerarsi irrealizzabile. Inoltre dovrebbero essere fissati degli intervalli di tempo massimo per le varie fasi ed un monitoraggio appropriato dovrebbe assicurare il rispetto degli stessi.

I principali Paesi donatori del GPAT sono gli Stati Uniti e la Svezia, ma, nonostante ciò, se consideriamo l'ammontare complessivo dei fondi statunitensi destinati a progetti contro la tratta di persone, il quantum ricevuto dal GPAT è piuttosto ridotto e la maggioranza dei progetti contro la tratta non afferenti al Programma Globale duplicano attività intraprese dal GPAT, a volte anche negli stessi Paesi o Regioni ${ }^{64}$.

Il ruolo dell'UNICRI all'interno del Programma Globale non è chiaramente definito, mentre sembra che l'Istituto abbia intenzione di mantenere l'autonoma capacità operativa recentemente sviluppata (due progetti anti-tratta sono stati completati e sei sono stati proposti). L'Istituto ha sede a Torino, è sostanzialmente finanziato dall'Italia e dunque la maggior parte dei progetti promossi non potrebbero essere messi in atto se non attraverso la collaborazione con il Governo italiano. Da ciò si ricava la raccomandazione di stabilire se l'UNICRI debba oggi considerarsi o meno partner del GPAT, perché nel primo caso i futuri progetti dell'Istituto, anche se approvati ed implementati dallo stesso, dovrebbero essere assorbiti nel Programma Globale.

Si raccomanda inoltre all'UNODC di esaminare gli accordi contrattuali con l'UNOPS e di rivalutare l'opportunità di servirsi di esso come implementing agency, considerando che gli eccessivi ritardi nei pagamenti di cui è incaricato hanno costretto, in alcuni casi, gli esperti internazionali ed i National Coordinators ad autofinanziarsi le missioni o le attività, in attesa di successivo rimborso.

In conclusione, il modello (best practice) di progetto di cooperazione tecnica, emerso dalle lessons learned ricavate dalla valutazione degli otto progetti già ricordati, dovrebbe includere le seguenti caratteristiche:

- un project design elaborato a livello locale in collaborazione con il Paese o la Regione beneficiari (con la supervisione dell'Headquarter di Vienna);

- una risposta pronta ed entusiasta del Governo locale;

\footnotetext{
${ }^{64} \mathrm{Nel}$ 2003, ad esempio, l'U.S. Trafficking in Persons Office ha finanziato duecentoventitrè progetti anti-tratta, di cui solo sei afferenti al Programma Globale, mentre tra quelli esterni al GPAT ventotto erano destinati a Paesi in cui il GPAT è pure presente. Si raccomanda, dunque, un rapporto più intenso di confronto e collaborazione dell'AHTU con l'U.S. Trafficking in Persons Office al fine di identificare le best practices e le priorità del donatore e di evitare duplicazioni di progetti.
} 
- un adeguato schema temporale per l'implementazione del progetto che assicuri l'indipendenza di ciascun elemento dall'altro, eliminando il vincolo ad un processo sequenziale troppo rigido che rischia di arrestare l'esecuzione dei progetti;

- la selezione del miglior partner (Agenzia, Ministero, Dipartimento) all'interno del Governo ospitante;

- l'identificazione e la selezione di un unico National Coordinator competente (ove ne sono stati nominati due, il progetto ha subito ritardi e difficoltà di gestione);

- la presenza di un Field Office Programme Manager competente e capace di supervisionare il progetto e di fornire assistenza al National Coordinator (in proposito sarebbe infatti opportuno rimandare l'implementazione dei progetti destinati ad aree in cui il Field Office non è ancora presente);

- un monitoraggio ed una valutazione costanti;

- un adeguato supporto dal Regional Desk al Field Office e dall'AHTU al Regional Desk ed al Field Office.

\section{Conclusioni}

Il punto focale del GPAT, come già visto in precedenza, è l'Anti-Human Trafficking Unit, il cui staff di base è composto permanentemente da due soli Professionals (cioè due sole persone che vengono pagate con il budget regolare delle Nazioni Unite ) ed occasionalmente (seppure oggi quasi con regolarità) da Associate Experts, Consultants ed esperti internazionali che sono pagati dai Paesi donatori nonché da interns e volunteers che sono selezionati per mansioni specifiche e prestano gratuitamente la propria collaborazione. Per quanto convinti che i risultati raggiunti fino ad oggi dal Programma Globale siano attribuibili all'alta competenza ed alla devozione al lavoro dello staff, crediamo che un Programma Globale che impiega solo due professionals sia da considerare (e talvolta è così considerato al di fuori dell'UNODC) un tentativo non sufficientemente adeguato delle Nazioni Unite di combattere una forma di crimine organizzato complessa quale la tratta di esseri umani. Ció comporta che, nella sua forma attuale, il GPAT è pressoché insostenibile poiché circa quaranta attività sono incluse nel work plan e lo staff esistente non può essere in grado di assolvere tutte le funzioni e rispettare tutte le scadenze. È dunque assolutamente urgente l'espansione dell'unità, soprattutto in considerazione del fatto che essa dovrebbe anche farsi carico di attività e progetti contro lo smuggling di migranti.

In secondo luogo, gli obiettivi ed i risultati del GPAT non sono noti se non ai Paesi donatori ed alle agenzie che si occupano di lotta contro la tratta di persone. Il sito web (<http://www.unodc.org/unodc/en/human-trafficking/index.html >) è il principale mezzo di diffusione delle notizie alla comunità internazionale ma, nonostante ciò, le informazioni in esso contenute sono spesso limitate, non aggiornate e descrivono solamente i risultati preliminari del Programma. Il Programma Globale non apporta un regolare contributo alle riviste accademiche a causa dell'eccessivo carico di lavoro pendente presso l'AHTU e di una politica molto restrittiva sui diritti della proprietà intellettuale; è infatti fatto divieto agli esperti internazionali di scrivere articoli su riviste accademiche o di condurre studi secondari utilizzando i dati e le informazioni appresi nel corso delle attività del programma. Ciò limita, da un lato, l'incidenza dei risultati delle attività del GPAT sulla letteratura in materia e, conseguentemente, sulle risposte contro il fenomeno che possono essere sviluppate a livello internazionale e, dall'altro, l'impatto che $\mathrm{i}$ «prodotti» del GPAT riescono 
ad avere sulla comunità dei donatori. Noi crediamo che trattandosi di un Programma finanziato pubblicamente, dovrebbe essere garantito l'accesso ai dati agli Stati membri e dovrebbe essere vinta la preoccupazione dei responsabili del Programma di veder pubblicamente criticate le mancanze dell'UNODC e dell'AHTU riguardo alla conduzione dei progetti di cooperazione tecnica e delle altre attività. Una soluzione potrebbe essere quella di prevedere delle pubblicazioni congiunte, cui prendano parte esperti internazionali esterni ed un global programme professional, in modo da far conoscere il punto di vista del Programma Globale sui rilievi più importanti e di contribuire alla disseminazione delle best pratices in materia. A tal proposito sarebbe opportuno che il GPAT creasse e mantenesse un database delle best practices poiché, in mancanza di un'adeguata e regolare riflessione sui progetti in corso e senza un confronto con i risultati raggiunti mediante l'intervento di altre organizzazioni, le soluzioni presentate dal GPAT come best practices rischiano di non risultare credibili.

I migliori risultati del GPAT sono stati raggiunti mediante $\mathrm{i}$ progetti di cooperazione tecnica e possono essere, a nostro avviso, valutati nella migliore maniera se prendiamo in considerazione: l'aumento di coscienza sul fenomeno di tratta nei Paesi destinatari dei progetti e negli Stati membri in generale; il miglioramento delle capacità delle forze di polizia e giudiziarie ottenuto mediante attività di training alle stesse; il numero di Stati che hanno ratificato il Protocollo sulla Tratta ${ }^{65}$; il numero di Stati che hanno sviluppato Piani d'Azione a livello nazionale; il numero di Stati che hanno approvato misure legislative specifiche sulla tratta; il numero di Stati che hanno rafforzato la cooperazione fra le agenzie governative impegnate nella lotta alla tratta ed altro ancora. Purtroppo l'assenza di statistiche e dati particolareggiati provenienti dai Paesi non ci consente di fornire un quadro completo della situazione attuale ma facendo riferimento ai dati riferiti ai Paesi coinvolti dai progetti che sono stati oggetto della valutazione da parte dell'IEU, possiamo comunque esprimere un giudizio, seppur limitato.

La sensibilizzazione dei giudici, dei pubblici ministeri e degli ufficiali di polizia è avvenuta mediante la realizzazione di presentazioni di esperti internazionali sull'impatto che la tratta ha sulle vittime ad esempio in Vietnam; il rafforzamento della cooperazione tra le agenzie governative è avvenuto mediante la creazione di inter-agency executive committees ad esempio nelle Filippine, in Repubblica Ceca ed in Colombia; l'identificazione di vuoti legislativi e la conseguente revisione della legislazione è avvenuta ad esempio in Polonia, Vietnam, Repubblica Ceca e Colombia; sessioni di training alle forze di polizia ed agli agenti

${ }^{65}$ Il Protocollo delle Nazioni Unite sulla tratta di persone è stato ratificato ad oggi da centosedici Stati. La maggior parte di essi è costituita da Paesi in via di sviluppo, nei quali la popolazione vive in stato di povertà o semipovertà. Alcuni Paesi industrializzati hanno ratificato il Protocollo: sono il Belgio, la Francia, la Russia, la Spagna, gli Stati Uniti e piú di recente la Gran Bretagna, la Germania e l'Italia. È evidente, ad ogni modo, la mancata ratifica di altri importanti Stati che rappresentano i principali Paesi di destinazione delle persone trafficate: la Cina (non firmatario), la Corea, il Giappone, la Grecia e su altro fronte, l'India, l'Indonesia, la Tailandia ed Israele. Se, infatti, è dato di pensare che i Paesi industrializzati siano dotati di una qualche normativa interna che vieta la schiavitù o quantomeno regola l'immigrazione clandestina, nei Paesi in via di sviluppo tali normative molte volte non esistono o vengono volutamente ignorate a causa della corruzione dilagante negli organi politici e governativi e nelle autorità preposte al contrasto del fenomeno; per questo motivo il Protocollo di Palermo contiene numerose disposizioni che mirano a facilitare l'attuazione del trattato per i Paesi in via di sviluppo.

Al fine di verificare l'effettiva utilità del Protocollo per gli Stati industrializzati, bisogna considerare, invece, un altro fondamentale obiettivo del trattato: quello di armonizzare le legislazioni degli Stati in materia, al fine di creare una rete di mezzi efficienti, e non in contrasto tra loro, che possano concretamente combattere il fenomeno. Qualora solamente i Paesi del Sud del mondo coordinassero i propri sforzi secondo i dettami del Protocollo e gli Stati del Nord attuassero di contro una cooperazione autonoma tra di loro con mezzi diversi, lo scopo di armonizzazione si vanificherebbe e tornerebbero a manifestarsi quei gravi problemi che fino all'attuazione del Protocollo hanno impedito un efficace e coordinato contrasto del fenomeno. 
di frontiera per migliorare la loro capacità investigativa sono state condotte ad esempio in Nigeria, Togo, Benin, Filippine e Vietnam. Inoltre i Governi beneficiari e le ONG hanno dichiarato che, senza la presenza del GPAT, nelle Filippine, in Vietnam ed in Polonia non sarebbero stati sviluppati i Piani d'Azione Nazionale; in Polonia, Repubblica Ceca e nelle Filippine non sarebbero state introdotte misure legislative anti-tratta ed infine che Benin, Polonia e Filippine non avrebbero ratificato il Protocollo sulla Tratta.

A parte Benin, Polonia e Filippine, su cui possiamo esprimerci con certezza, altri Paesi hanno beneficiato di progetti di pre-ratification assistance del GPAT e precisamente Brasile, Colombia, Libano, Moldova, Slovacchia e sei Paesi della Southern African Development Community (Botswana, Lesotho, Malawi, Namibia, Sud Africa e Zambia), ma non siamo in grado di dire esattamente per quanti e quali la presenza del GPAT sia stata determinante per la ratifica. A prescindere da ciò, non condividiamo interamente il punto di vista di alcuni membri del Programma Globale che ritengono che la ratifica del Protocollo sulla Tratta rappresenti un punto d'arrivo dell'intervento delle Nazioni Unite, essendo convinti che questa non sia da sé sufficiente ad assicurare l'adempimento delle obbligazioni da parte degli Stati membri. È vero che la ratifica garantisce l'ingresso degli Stati membri nella Conference of the Parties to the Transnational Organized Crime Convention, che ha ricevuto un mandato dall'Assemblea Generale per assicurare l'implementazione dei Protocolli annessi alla Convenzione mediante l'assistenza tecnica ai Paesi, ma è anche vero che diversi Stati hanno manifestato la propria incapacità di adempiere alle obbligazioni derivanti dal Protocollo sulla Tratta ed hanno richiesto un'assistenza più lunga ed accurata. Per tale ragione crediamo che, potendo considerarsi conclusa la fase dimostrativa dei progetti (iniziata con il lancio del programma nel I999), il GPAT debba adesso estendere il suo intervento anche alla fase di post-ratificazione.

Ai risultati certamente soddisfacenti nel campo dei progetti di assistenza tecnica si accompagnano risultati inadeguati nel campo della ricerca e soprattutto della raccolta dati. Se l'UNODC individua il mandato del Programma Globale nel Protocollo per Prevenire, Eliminare e Punire la tratta di persone, per valutare gli obiettivi raggiunti tramite il GPAT dovremmo poter dare risposta ai seguenti quesiti: il Programma Globale ha contribuito a prevenire ed eliminare il fenomeno della tratta di persone? Il Programma Globale ha contribuito a far condannare i trafficanti? Il Programma Globale ha contribuito ad offrire supporto e protezione alle vittime ed ai testimoni di tratta?

La mancanza di dati completi ed affidabili che misurino l'incidenza del fenomeno di tratta nonché l'eventuale diminuzione del numero di persone trafficate ed aumento del numero di trafficanti condannati non consente, nemmeno agli osservatori esterni ed agli stessi evaluators, di dare risposta a tali quesiti e dunque di determinare se il GPAT ha raggiunto o meno i suoi principali obiettivi nel campo della giustizia penale. Fino all'aprile del 2006, mese di pubblicazione del Report «Trafficking in Persons: Global Patterns» ${ }^{66}$ da parte dell'AHTU, l'United States Trafficking in Persons Report ha rappresentato il punto di riferimento in tal senso. Inoltre, il ritardo nel fornire questi dati ha reso vana la verifica richiesta ai sensi della Vienna Declaration on Crime and Justice $e^{67}$ la quale aveva fissato il 2005 come anno per valutare la diminuzione d'incidenza dei crimini transnazionali organizzati, tra cui la tratta di persone ed ha forse avuto un impatto negativo sui Paesi donatori, sui Governi beneficiari dei progetti, sulle organizzazioni governative e non governative, che talvolta hanno ritenuto preferibile il lavoro di altre entità o hanno puntato sul finanziamento di progetti al di fuori del GPAT.

\footnotetext{
${ }^{66}$ UNODC, Trafficking in Persons: Global Patterns, april 2006,

$<$ http://www.unodc.org/pdf/traffickinginpersons_report_20o6ver2.pdf> [consultato:03-I2-2007].

${ }^{67}$ Assemblea Generale delle NAZioni Unite, Risoluzione 55/59 del I7 gennaio 200I, «Vienna Declaration on Crime and Justice» in UN Official Records of the General Assembly, 55th sess, agenda item 105 (A/RES/55/59).
} 
Per quanto riguarda, infine, la sostenibilità dei risultati ottenuti, se consideriamo tali la ratifica del Protocollo sulla Tratta e l'adozione di misure legislative specifiche antitratta, possiamo affermare che essi sono durevolmente sostenibili, presumendo che le nuove norme introdotte non verranno successivamente abrogate e così pure l'elaborazione di training manuals costituisce un risultato sostenibile dopo il completamento del progetto nel Paese beneficiario. Se facciamo riferimento, invece, agli altri risultati, come già evidenziato, essi non sono sostenibili in presenza di uno staff così limitato e considerata la necessità di espandere il raggio d'azione del Programma Globale.

Vogliamo allora ricordare che il Programma Globale conta sull'appoggio di tutti gli Stati membri delle Nazioni Unite, secondo l'impegno assunto con la già ricordata Dichiarazione di Vienna:

«We reaffirm our continued support for and commitment to the United Nations Crime Prevention and Criminal Justice Programme, especially the Commission on Crime Prevention and Criminal Justice and the United Nations Centre for International Crime Prevention, the United Nations Interregional Crime and Justice Research Institute and the institutes of the Programme network, and resolve to strengthen the Programme further through sustained funding, as appropriate».

Fino ad oggi, solo pochi Paesi (tra cui Francia, Germania e Giappone) hanno finanziato posizioni di Associate Experts all'interno dell'AHTU, solo sei Paesi (Austria, Germania, Italia, Olanda, Svezia e Stati Uniti) hanno contribuito al Programma con fondi non specificamente indirizzati e solo sedici Paesi hanno finanziato progetti specifici. Si auspica, dunque, che l'impegno degli Stati membri sia rafforzato, in modo da consentire la programmazione di iniziative a lunga scadenza ed il miglioramento delle componenti del GPAT di cui abbiamo messo in evidenza i punti ancora deboli. 